\title{
Water-Soluble Pentagonal-Prismatic Titanium-Oxo Clusters
}

Guanyun Zhang, ${ }^{a}$ Caiyun Liu, ${ }^{\mathrm{a}}$ De-Liang Long, ${ }^{\mathrm{b}}$ Leroy Cronin, ${ }^{\mathrm{b}}$ Chen-Ho Tung ${ }^{\mathrm{a}}$ and Yifeng Wang*a

${ }^{a}$ Key Lab for Colloid and Interface Science of Ministry of Education, School of Chemistry and Chemical Engineering, Shandong University, Ji'Nan 250100, P. R. China; ${ }^{b}$ WestChem, School of Chemistry, University of Glasgow, Glasgow G12 8QQ, United Kingdom

\section{Table of contents}

Section S1. Syntheses and methods

S1.1 Materials

S1.2 Syntheses

S1.3 Methods

S1.4 Single crystal X-ray crystallography

Section S2. Structure and analysis

Table S1. Selected bond parameters and bond valence calculations for 1a

Figure S1. The two enantiomers of $\mathbf{1 a}$

Figure S2. Negative mode ESI-MS of 1

Table S2. Peak assignments for negative mode ESI-MS

Figure S3. Positive mode ESI-MS of 1

Table S3. Peak assignments for positive mode ESI-MS

Figure S4. ${ }^{17} \mathrm{O}$ NMR of ${ }^{17} \mathrm{O}$-enriched $\mathrm{CH}_{3} \mathrm{COOH}$ and $\mathrm{SO}_{4}{ }^{2-}$

Section S3. Vibrational spectra

Figure S5. IR and Raman spectra of 1

Figure S6. IR spectra of $\mathbf{1}$ in $\mathrm{MeCN}$

Figure S7 - S9. IR spectra of 1 in water

Figure S10. IR spectra of $\mathbf{1}$ in methanol, nitromethane, THF and acetone

Figure S11. IR of the air-dried residue of the aged solution samples

Figure S12. IR of 2 in $1.0 \mathrm{M} \mathrm{HCl}, \mathrm{MeCN}$ and acetone

Figure S13. IR of 3 in various solutions

Figure S14. IR of 4 in various solutions

Figure S15. TGA analysis of 1

Section S4. Preparation of $\mathbf{1 / G O}$ and more TEM images

Section S5. UV-vis absorption and diffuse-reflectance spectra

Figure S16. UV-vis absorption spectra of 1 in $\mathrm{MeCN}$

Figure S17. Diffuse reflectance spectra of compounds 1, 2 and 3

Section S6. Photocurrent

Figure S18. Photocurrent data and IR of the samples

Section S7. Photocatalytic benzyl alcohol oxidation

Figure S19. Photocatalytic benzyl alcohol oxidation

Figure S20. IR spectra of $\mathbf{1}$ in benzyl alcohol

Figure S21. Time-resolved solution IR during photocatalytic oxidation of benzyl alcohol 


\section{Section S1. Synthesis and Methods}

S1.1 Materials. All the chemicals were of analytical grade, obtained from commercial sources and used as received.

\section{S1.2 Syntheses}

Caution: Dissolution of $\mathrm{TiCl}_{4}$ in water emits lots of heat and corrosive acidic moisture and thus must be performed in a well-ventilated fume hood, meanwhile gloves, safety goggles and face shields should be worn. Water should be added slowly and in portions while an ice-cold water bath should be used to cool the solution.

$\left[\mathrm{Ti}_{18} \mathrm{O}_{27}\left(\mathrm{OH}_{2}\right)_{30}\left(\mathrm{SO}_{4}\right)_{6}\right] \mathrm{Cl} 6 \cdot 6 \mathrm{TBAC} \cdot 12 \mathrm{H}_{2} \mathrm{O}(\mathbf{1})$. A mixture of $\mathrm{TiCl}_{4}(1.0 \mathrm{M} ; 10 \mathrm{~mL}), \mathrm{H}_{2} \mathrm{SO}_{4}(0.33 \mathrm{M})$ and tetrabutylammonium chloride (TBAC; $1.0 \mathrm{M}$ ) was stirred for $24 \mathrm{~h}$ under ambient conditions. After filtration, slow evaporation of the filtrate afforded colorless needle-like crystals of compound $\mathbf{1}$ in three weeks in ca. 69\% yield (based on Ti and a $50 \mathrm{~mL}$ reaction). The product was collected by filtration and air-dried with suction for 10 minutes. Anal. Calcd (\%): Ti, 19.13; C, 25.57; N, 1.86; H, 6.66; Cl, 9.46. Found (\%): Ti, 19.73; C, 25.69; N, $1.87 ; \mathrm{H}, 6.65 ; \mathrm{Cl}, 9.57$.

${ }^{17} \mathrm{O}$-enriched 1 . The procedure is the same as the above description, except that $10 \%{ }^{17} \mathrm{O}$-enriched water was used. The content of ${ }^{17} \mathrm{O}$ in the as-prepared 1 should be less than $10 \%$, due to using of normal $\mathrm{H}_{2} \mathrm{SO}_{4} .10 \%$ ${ }^{17} \mathrm{O}$-enriched water was used to dissolve $\mathbf{1}$ for acquiring the NMR measurements.

${ }^{18} \mathrm{O}$-enriched 1 . The procedure is the same as the above description, except that $97 \%{ }^{18} \mathrm{O}$-enriched water was used. The content of ${ }^{18} \mathrm{O}$ in the as-prepared $\mathbf{1}$ is estimated to be ca. $92 \%$. This sample was used to study the vibrational spectra.

$\left[\mathrm{Ti}_{18} \mathrm{O}_{27}\left(\mathrm{OH}_{2}\right)_{31}\left(\mathrm{SO}_{4}\right)_{6}\right]_{2}\left[\mathrm{Ti}_{18} \mathrm{O}_{27}\left(\mathrm{OH}_{2}\right)_{28}\left(\mathrm{SO}_{4}\right)_{8}\right]\left(\mathrm{HSO}_{4}\right)_{6} \mathrm{Cl}_{8} \cdot 15 \mathrm{TBAC} \cdot 2 \mathrm{OH} \mathrm{H}_{2} \mathrm{O}(2)$. The protocol is the same as that for compound 1, except that concentration of $\mathrm{H}_{2} \mathrm{SO}_{4}$ was $1.0 \mathrm{M}$. Yield is $67 \%$, based on Ti and a $40 \mathrm{~mL}$ reaction. Anal. Calcd (\%): Ti, 20.16; C, 22.46; N, 1.64; H, 5.98; Cl, 6.37. Found (\%): Ti, 20.26; C, 22.30; N, $1.71 ; \mathrm{H}, 5.87 ; \mathrm{Cl}, 6.47$.

$\left[\mathrm{Ti}_{18} \mathrm{O}_{27}\left(\mathrm{OH}_{2}\right)_{26}\left(\mathrm{SO}_{4}\right)_{9}\right] \cdot 7 \mathrm{TBAC} \cdot 8 \mathrm{H}_{2} \mathrm{O}(\mathbf{3})$. The protocol is the same as that for compound 1, except that (TBA) ${ }_{2} \mathrm{SO}_{4}(2.0 \mathrm{M})$ was used instead of $\mathrm{H}_{2} \mathrm{SO}_{4}$ and TBAC. Yield is ca. $70 \%$, based on Ti and a $20 \mathrm{~mL}$ reaction. Anal. Calcd (\%): Ti, 18.28; C, 28.51; N, 2.08; H, 6.79; Cl, 5.27. Found (\%): Ti, 18.87; C, 28.70; N, 2.32; H, 6.81; $\mathrm{Cl}, 5.58$.

$\left[\mathrm{Ti}_{18} \mathrm{O}_{27}\left(\mathrm{OH}_{2}\right)_{24}\left(\mathrm{SeO}_{4}\right)_{9}\right] \cdot 6 \mathrm{TBAC} \cdot 2 \mathrm{HCl} \cdot 24 \mathrm{H}_{2} \mathrm{O}(4)$. The protocol is the same as that for compound $\mathbf{1}$, except that $0.4 \mathrm{M} \mathrm{H}_{2} \mathrm{SeO}_{4}$ was used instead of $\mathrm{H}_{2} \mathrm{SO}_{4}$. Yield is ca. $26 \%$ based on $\mathrm{Se}$ and a $10 \mathrm{~mL}$ reaction. Anal. Calcd (\%): Ti, 16.61; C, 22.21; N, 1.62; H, 6.05; Cl, 5.48. Found (\%): Ti, 16.68; C, 19.6; N, 1.62; H, 5.15; Cl, 5.57.

${ }^{17} \mathrm{O}$-enriched $\mathrm{CH}_{3} \mathrm{COOH} .{ }^{17} \mathrm{O}$-enriched $\mathrm{CH}_{3} \mathrm{COOH}$ was synthesized via quantitative reaction of $\mathrm{CH}_{3} \mathrm{COCl}$ with ${ }^{17} \mathrm{O}$-enriched water (ca. 10 atom $\%{ }^{17} \mathrm{O}$ ) in $\mathrm{MeCN}$. The resultant solution was sealed in a Wilmad® coaxial insert as an external standard of ${ }^{17} \mathrm{O}$ NMR to calibrate the chemical shift under different conditions.

${ }^{17}$ O-enriched $\mathrm{H}_{2} \mathrm{SO}_{4}$. Concentrated $\mathrm{H}_{2} \mathrm{SO}_{4}(18 \mathrm{M})$ was dissolved in ${ }^{17} \mathrm{O}$-enriched water. This was used to understand the ${ }^{17} \mathrm{O}$ NMR of free $\mathrm{SO}_{4}{ }^{2-}$ ions.

S1.3 Methods. Single crystal X-ray diffraction data were collected using a Bruker SMART APEX II diffractometer equipped with a CCD area detector using Mo K $\alpha$ radiation $(\lambda=0.71073 \AA$ ). Powder X-ray diffraction (PXRD) measurements were performed on a Bruker D8 Advance X-ray diffraction instrument using 
$\mathrm{Cu}-\mathrm{K} \alpha$ radiation. All the IR spectra were measured using the attenuated total reflection Fourier transformation infrared spectroscopy (ATR-FTIR; PerkinElmer Spectrum Two FT-IR). Raman spectra were obtained on a NEXUS 670 FT-IR Raman spectrometer with $473 \mathrm{~nm}$ excitation. Elemental analyses (C, H, and N) were measured on a FLASH EA1112 elemental analyzer. Content of Ti was determined using a colorimetric method as described previously. ${ }^{1}$ Content of $\mathrm{Cl}$ was determined using argentometry based on the Mohr method. ${ }^{17} \mathrm{O}$ NMR was recorded using the Bruker $400 \mathrm{MHz}$ Fourier transform NMR spectrometer in $5 \mathrm{~mm}$ NMR tubes. Transmission electron microscopy (TEM) was carried out using the JEM-2010F instrument. Atomic force microscopic (AFM) images were acquired on a Dimension Icon Scanning Probe Microscope (SPM; Veeco Instruments Inc). X-ray photoelectron spectroscopy (XPS) was performed using the Perkin-Elmer PHI5300 instrument. Mass Spectrometry measurements for all compounds were performed on a Waters Synapt-G2 instrument equipped with Quadrupole and Time of Flight modules (Q/TOF). The standard tuning mix used allowed calibration between $500-5000 \mathrm{~m} / \mathrm{z}$ and the samples were introduced using a Harvard syringe pump. Typical settings were: a capillary voltage of $2.75 \mathrm{kV}$, a sampling cone voltage of $35 \mathrm{kV}$, an extraction cone voltage of $4.0 \mathrm{kV}$, a source temperature of $80^{\circ} \mathrm{C}$, a desolvation temperature of $180^{\circ} \mathrm{C}$, a cone gas flow of $15 \mathrm{~L}$ $\mathrm{h}^{-1}$ and a desolvation gas flow of $750 \mathrm{~L} \mathrm{~h}^{-1}$.

S1.4 Single crystal X-ray crystallography. Single crystals of appropriate dimensions were chosen from the mother liquor with the help of an optical microscope, coated with high vacuum grease (Dow Corning Corporation), and transferred to the diffractometer. Data were collected at $173 \mathrm{~K}$. Structure solution and refinement were performed using the ShelXL package and the least-squares method implemented by Olex2 v1.2. ${ }^{2}$ Hydrogen atoms were added theoretically. Since crystal quality of compounds $\mathbf{2}$ and $\mathbf{3}$ are not as good as compound 1, isotropic refinement was not performed to some atoms. Crystallographic data and the powder Xray diffraction data are provided immediately below.

Comments for 2. Because of disorder of the solvent $\left(\mathrm{H}_{2} \mathrm{O}\right)$ and TBA ions which could not be overcome by other techniques (like acquiring data at $173 \mathrm{~K}$ ), "squeeze" (the Platon package ${ }^{3}$ ) was applied after the structure solving had been finished completely. Before "squeeze", R1 was 12.2. Then "squeeze" gave a residue electron density of $944 \mathrm{e}$ per cell. According to the elemental analysis results, we therefore added four TBAC molecules to each unit cell ( $4 \times 156$ e). Further refinements gave a better R1 value of $10.6 \%$.

Comments for 3. Before "squeeze", the R1 was 10.2\%. "Squeeze" gave a residue electron density of 432e per cell. Based on the elemental analysis results, we therefore added four TBAC molecules to each unit cell (4×156 e). Further refinements gave a better R1 value of $8.19 \%$.

Comments for 4. Before "squeeze", the R1 was $8.64 \%$. "Squeeze" gave a residue electron density of 284e per cell. Based on the elemental analysis results, we therefore added two TBAC to each unit cell $(2 \times 156 \mathrm{e})$. Further refinements gave a better R1 value of $7.13 \%$.

More comments on 2 and 3. 2 crystallizes in monoclinic system with a long crystallographic c-axis of $82.048 \AA$ (space group C2/c), which means that there are eight equivalent points in one unit cell. The asymmetric unit consists of a half cluster of $\mathbf{2} \mathbf{a}\left(\mathrm{H}_{56} \mathrm{O}_{87} \mathrm{~S}_{8} \mathrm{Ti}_{18}\right)$ and one cluster of $\mathbf{2} \mathbf{b}\left(\mathrm{H}_{62} \mathrm{O}_{82} \mathrm{~S}_{6} \mathrm{Ti}_{18}\right)$ which alternatively pack

1. Zhang, G.; Hou, J.; Tung, C.-H.; Wang, Y., Small Titanium Oxo Clusters: Primary Structures of Titanium(IV) in Water. Inorg. Chem. 2016, 55, 3212-3214.

2. Dolomanov, O. V.; Bourhis, L. J.; Gildea, R. J.; Howard, J. A. K.; Puschmann, H., OLEX2: a complete structure solution, refinement and analysis program. 2009, 42, 339-341.

3. (a) Spek, A.L. Acta Cryst. 2015 C71, 9-18.; (b) Spek, A.L. J. Appl. Cryst. 2003, 36, 7-13. 
along the c-axis and make the c-axis unusually long. As $\mathbf{2 a}$ (with eight $\mathrm{SO}_{4}$ ligands) and $\mathbf{2} \mathbf{b}$ (with six $\mathrm{SO}_{4}$ ligands) are unique and are significantly different from each other, no smaller unit cell of reduced c-axis exists. In fact, the X-ray diffraction was repeated using different batches of samples but the results were the same. Therefore the unit cell of $\mathbf{2}$ is not a "supercell" but real "primitive" cell. Also no twin issue was found. $\mathbf{3}$ crystallizes in orthorhombic system (space group P $2{ }_{1} 2_{1} 2_{1}$ ) with a long crystallographic c-axis of $60.828 \AA$, which means there are four equivalent points in every unit cell. The asymmetric unit consists of one cluster of $\mathbf{3 a}\left(\mathrm{H}_{52} \mathrm{O}_{89} \mathrm{~S}_{9} \mathrm{Ti}_{18}\right)$ together with some TBA cations, chloride anions and solvent water molecules. The X-ray diffraction was repeated and the data were carefully checked; no additional symmetry with smaller unit cell was found for this structure. However a little racemic twin was identified and the structure was refined with TWIN and BASF instructions. The resultant Flack factor 0.06(5) indicates the second twin component is minor and the chiral space group assignment is correct.

The following table summarizes the crystallographic data of compounds 1-4:

\begin{tabular}{|c|c|c|c|c|}
\hline Compound & 1 & 2 & 3 & 4 \\
\hline Formula unit & $\mathrm{C}_{96} \mathrm{H}_{300} \mathrm{Cl}_{12} \mathrm{~N}_{6} \mathrm{O}_{93} \mathrm{~S}_{6} \mathrm{Ti}_{18}$ & $\mathrm{C}_{240} \mathrm{H}_{766} \mathrm{Cl}_{23} \mathrm{~N}_{15} \mathrm{O}_{295} \mathrm{~S}_{26} \mathrm{Ti}_{54}$ & $\mathrm{C}_{112} \mathrm{H}_{320} \mathrm{Cl}_{7} \mathrm{~N}_{7} \mathrm{O}_{97} \mathrm{~S}_{9} \mathrm{Ti}_{18}$ & $\mathrm{C}_{96} \mathrm{H}_{314} \mathrm{Cl}_{8} \mathrm{~N}_{6} \mathrm{O}_{111} \mathrm{Se}_{9} \mathrm{Ti}_{18}$ \\
\hline Moieties & $\begin{array}{l}{\left[\mathrm{Ti}_{18} \mathrm{O}_{27}\left(\mathrm{OH}_{2}\right)_{30}\left(\mathrm{SO}_{4}\right)_{6}\right] \mathrm{Cl}_{6}} \\
\cdot 6 \mathrm{TBAC} \cdot 12 \mathrm{H}_{2} \mathrm{O}\end{array}$ & $\begin{array}{l}{\left[\mathrm{Ti}_{18} \mathrm{O}_{27}\left(\mathrm{OH}_{2}\right)_{31}\left(\mathrm{SO}_{4}\right)_{6}\right]_{2}} \\
{\left[\mathrm{Ti}_{18} \mathrm{O}_{27}\left(\mathrm{OH}_{2}\right)_{28}\left(\mathrm{SO}_{4}\right)_{8}\right]} \\
\left(\mathrm{HSO}_{4}\right)_{6} \mathrm{Cl}_{8} \cdot 15 \mathrm{TBAC} \cdot 20 \mathrm{H}_{2} \mathrm{O}\end{array}$ & $\begin{array}{l}{\left[\mathrm{Ti}_{18} \mathrm{O}_{27}\left(\mathrm{OH}_{2}\right)_{26}\left(\mathrm{SO}_{4}\right)_{9}\right]} \\
\cdot 7 \mathrm{TBAC} \cdot 8 \mathrm{H}_{2} \mathrm{O}\end{array}$ & $\begin{array}{l}{\left[\mathrm{Ti}_{18} \mathrm{O}_{27}\left(\mathrm{OH}_{2}\right)_{24}\left(\mathrm{SeO}_{4}\right)_{9}\right]} \\
\cdot 6 \mathrm{TBAC} \cdot 2 \mathrm{HCl} \cdot 24 \mathrm{H}_{2} \mathrm{O}\end{array}$ \\
\hline F. W. $(\mathrm{g} / \mathrm{mol})$ & 4505.286 & 12820.14 & 4714.066 & 5185.95 \\
\hline Crystal system & triclinic & monoclinic & orthorhombic & monoclinic \\
\hline Space group & $\mathrm{P}-1$ & $\mathrm{C} 2 / \mathrm{c}$ & $\mathrm{P} 2{ }_{1} 2_{1} 2_{1}$ & $\mathrm{P} 2{ }_{1} / \mathrm{m}$ \\
\hline $\mathrm{a}(\AA)$ & $18.2281(7)$ & $20.732(4)$ & $17.555(4)$ & $19.755(2)$ \\
\hline $\mathrm{b}(\AA)$ & 18.9491(8) & $31.885(6)$ & $18.035(3)$ & $28.773(3)$ \\
\hline c $(\AA)$ & $32.1353(13)$ & $82.048(16)$ & $60.828(12)$ & $20.175(2)$ \\
\hline$\alpha\left(^{\circ}\right)$ & $73.959(2)$ & 90 & 90 & 90 \\
\hline$\beta\left(^{\circ}\right)$ & $87.124(2)$ & 90.63 & 90 & $116.7520(10)$ \\
\hline$\delta\left({ }^{\circ}\right)$ & $76.941(2)$ & 90 & 90 & 90 \\
\hline Volume $\left(\AA^{3}\right)$ & $10390.8(7)$ & $54127(19)$ & $19258(7)$ & $10240(2)$ \\
\hline Z & 2 & 4 & 4 & 2 \\
\hline Density $_{\text {calc }}\left(\mathrm{g} / \mathrm{cm}^{3}\right)$ & 1.433 & 1.573 & 1.627 & 1.682 \\
\hline $\begin{array}{l}\text { Abs. Coeff. } \mu \\
\left(\mathrm{mm}^{-1}\right)\end{array}$ & 0.947 & 1.057 & 0.993 & 2.459 \\
\hline Temperature (K) & 173.15 & 173.15 & 173.15 & 173.15 \\
\hline Total reflections & 141932 & 163602 & 37221 & 53408 \\
\hline Min-max $2 \theta\left(^{\circ}\right)$ & 4.99 to 50 & 1.99 to 50.712 & 4.83 to 49.992 & 4.94 to 54.93 \\
\hline Unique reflections & 36560 & 48657 & 17804 & 23737 \\
\hline $\mathrm{R}_{\text {int }}$ & 0.0950 & 0.0514 & 0.0554 & 0.0582 \\
\hline $\begin{array}{l}\text { Calculated } \\
\text { reflection }(I>2 \sigma)\end{array}$ & 17583 & 42304 & 12830 & 11867 \\
\hline $\mathrm{R}_{1}[\mathrm{I}>=2 \sigma]$ & 0.1157 & 0.1063 & 0.0819 & 0.0713 \\
\hline $\mathrm{wR}_{2}$ (all data) & 0.3726 & 0.3053 & 0.2107 & 0.2172 \\
\hline $\begin{array}{l}\text { Goodness of fit on } \\
\mathrm{F}^{2}\end{array}$ & 1.162 & 1.094 & 1.048 & 0.974 \\
\hline Parameters & 2076 & 2489 & 1329 & 1115 \\
\hline Restraints & 220 & 105 & 175 & 120 \\
\hline $\begin{array}{l}\text { Largest diff. } \\
\text { peak/hole }\left(e \AA^{-3}\right)\end{array}$ & $2.34 /-1.56$ & $2.61 /-1.49$ & $1.79 /-0.62$ & $2.43 /-1.75$ \\
\hline
\end{tabular}


The following figures are the experimental and the simulated PXRD data:
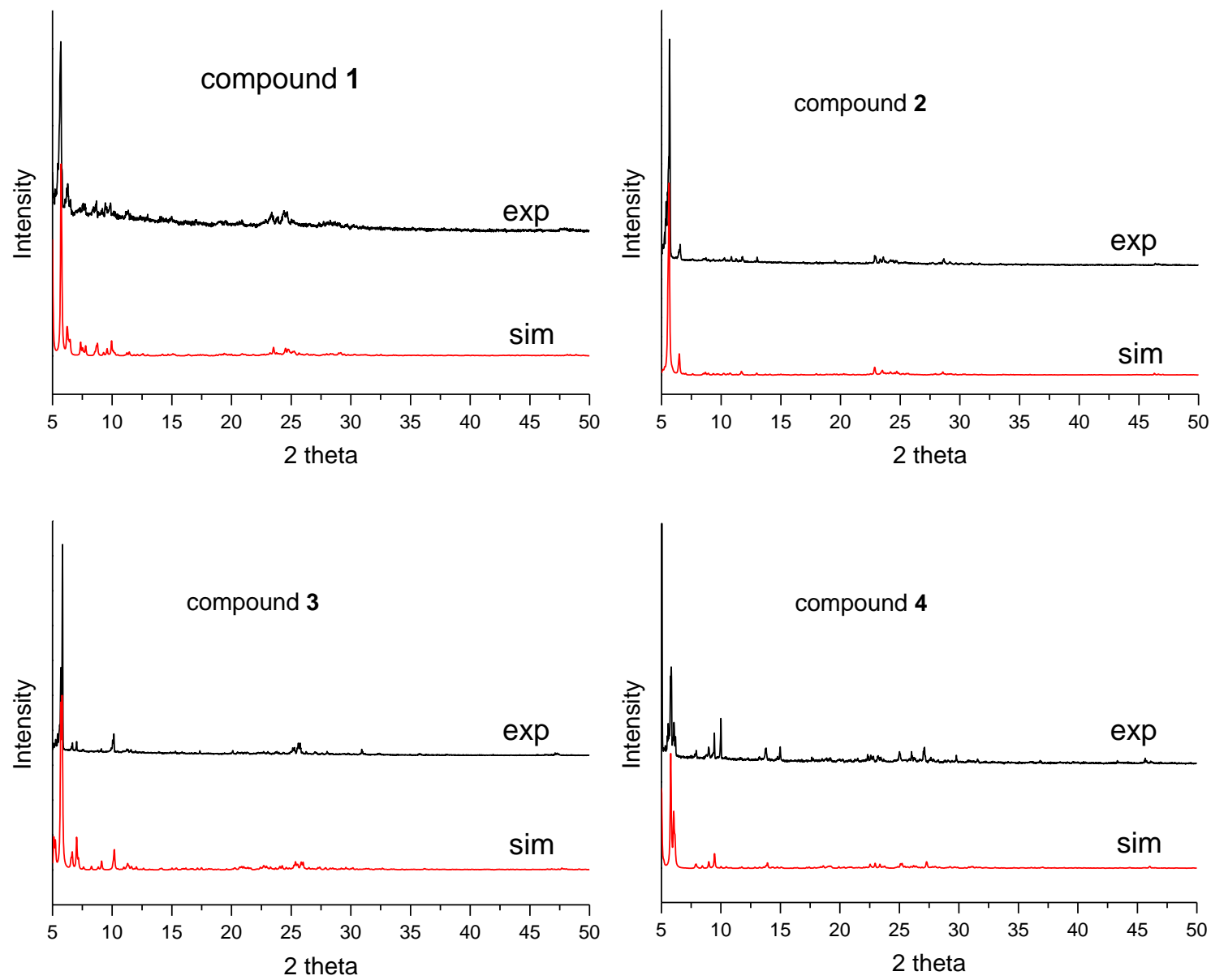

Section S2. Structures and Analysis

Table S1. Selected bond parameters and bond valence calculations ${ }^{\text {a }}$ for cluster 1a

\begin{tabular}{|c|c|c|}
\hline cluster 1a & & \\
\hline Ti-O bonds & Bond lengths, Å & Bond valence calculations \\
\hline Ti-O(aqua) & $2.057-2.242$ & $0.520-0.315$ \\
\hline $\mathrm{Ti}-\left(\mu_{2}-\mathrm{O}\right)$ & $1.704-1.943$ & $1.350-0.708$ \\
\hline $\operatorname{Ti}($ apex $)-\left(\mu_{3}-\mathrm{O}\right)$ & $1.852-1.930$ & $0.905-0.733$ \\
\hline $\mathrm{Ti}($ center $)-\left(\mu_{3}-\mathrm{O}\right)$ & $2.018-2.161$ & $0.578-0.393$ \\
\hline $\mathrm{Ti}-\mathrm{O}\left(\mathrm{SO}_{4}\right)$ & $2.005-2.088$ & $0.598-0.478$ \\
\hline \multicolumn{3}{|l|}{ Ti-O-Ti bond angles, ${ }^{\circ}$} \\
\hline $\mathrm{Ti}($ apex $)-\left(\mu_{3}-\mathrm{O}\right)-\mathrm{Ti}($ apex $)$ & $148.66-154.98$ & \\
\hline $\operatorname{Ti}($ apex $)-\left(\mu_{3}-\mathrm{O}\right)-\mathrm{Ti}($ center $)$ & $99.96-106.01$ & \\
\hline $\operatorname{Ti}($ center $)-\left(\mu_{2}-\mathrm{O}\right)-\mathrm{Ti}($ center $)$ & $178.35-179.37$ & \\
\hline $\operatorname{Ti}($ apex $)-\left(\mu_{2}-\mathrm{O}\right)-\mathrm{Ti}($ apex $)$ & $159.49-162.51$ & \\
\hline
\end{tabular}

a Bond valence calculations were performed using Bond Valence Calculator (Version 2.0, February 1993), and default parameters provided by Bond Valence Calculator were used, i.e., $R_{0}=1.815$ and $B=0.370$. 


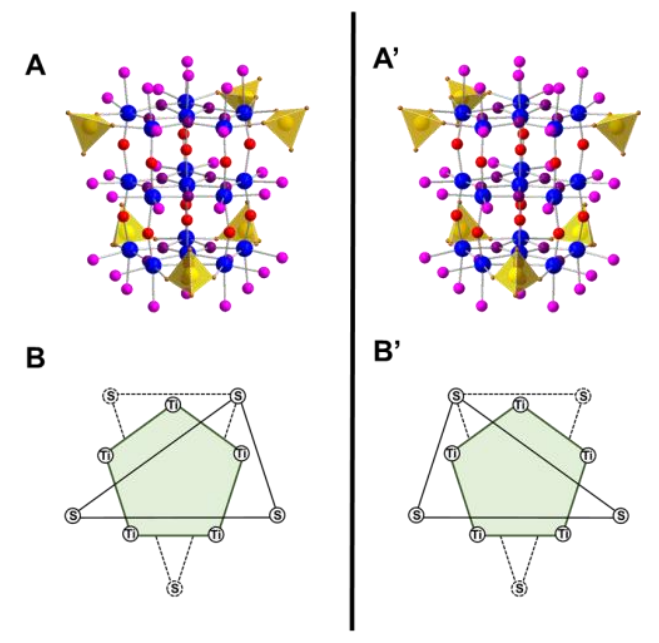

Figure S1. The two enantiomers of 1a: (A and $\left.\mathbf{A}^{\prime}\right)$ the side views and ( $\mathbf{B}$ and $\left.\mathbf{B}^{\prime}\right)$ cartoon illustration of the top views.

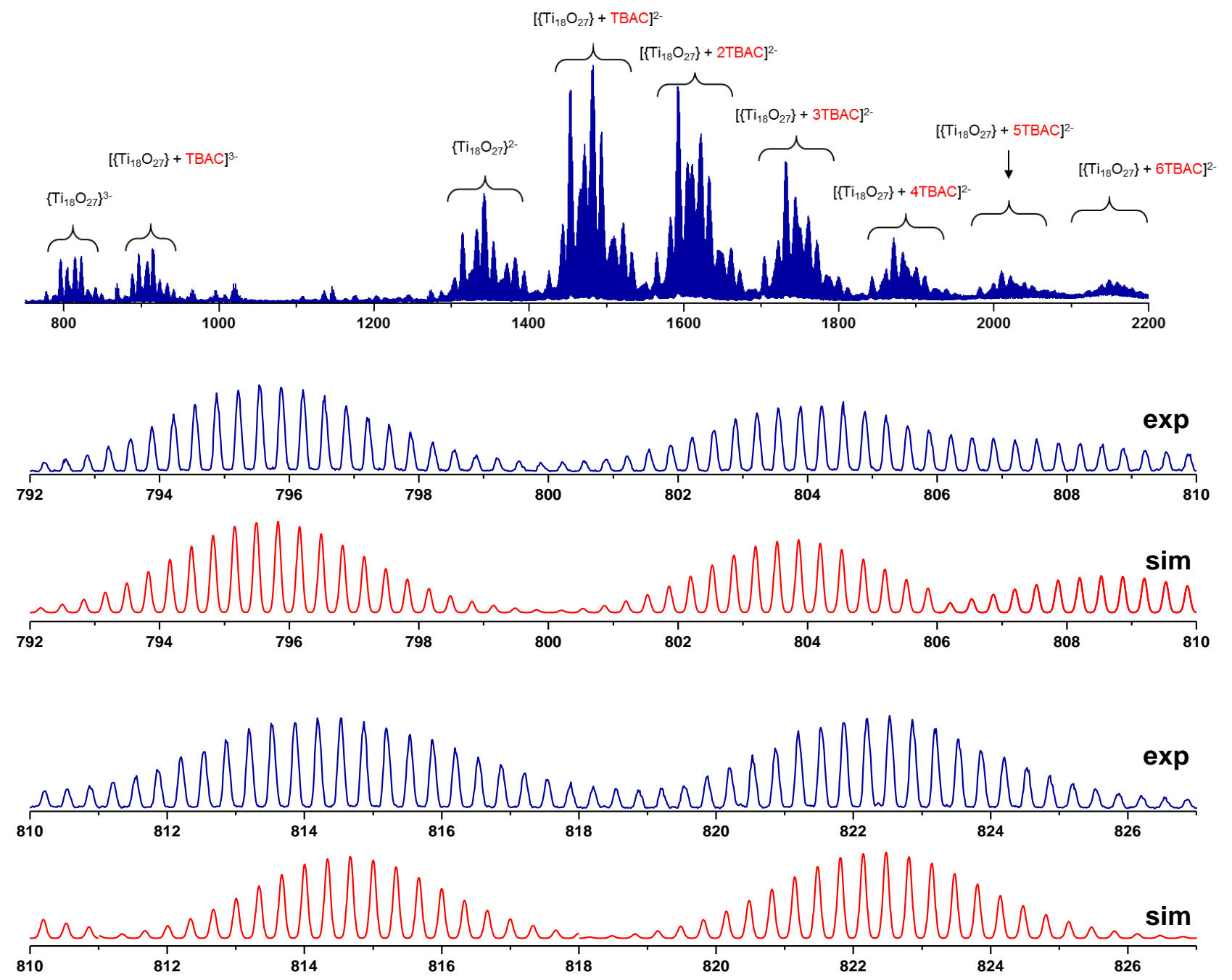



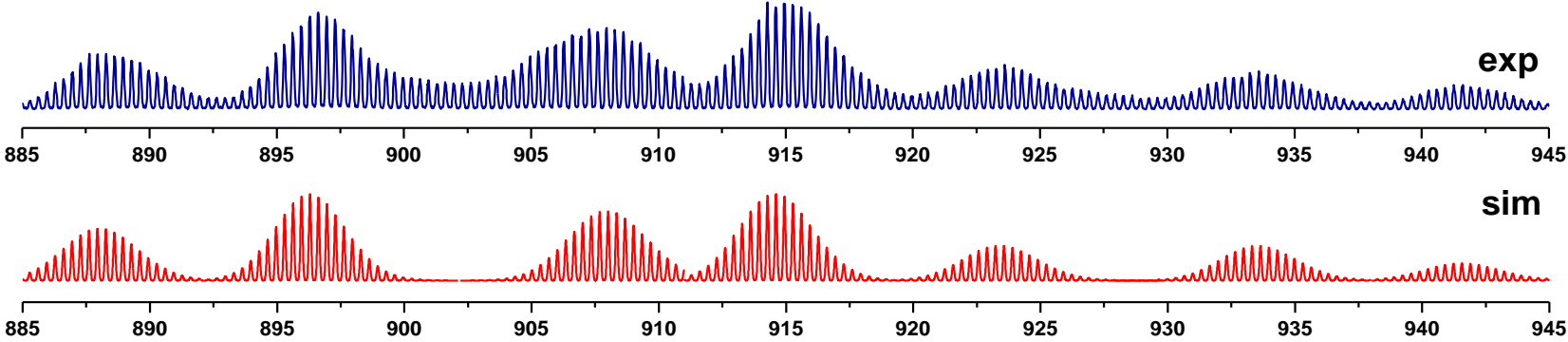

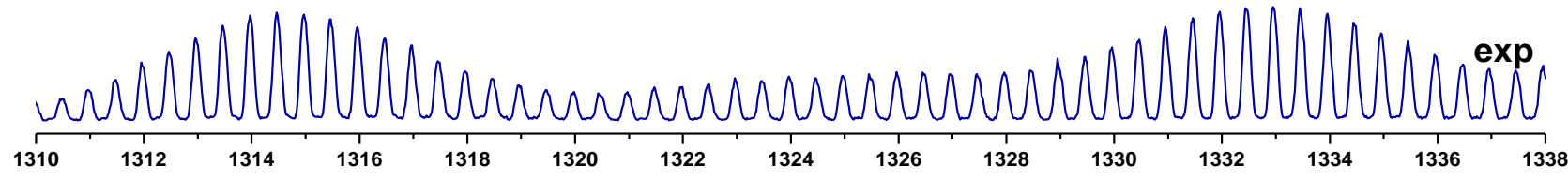

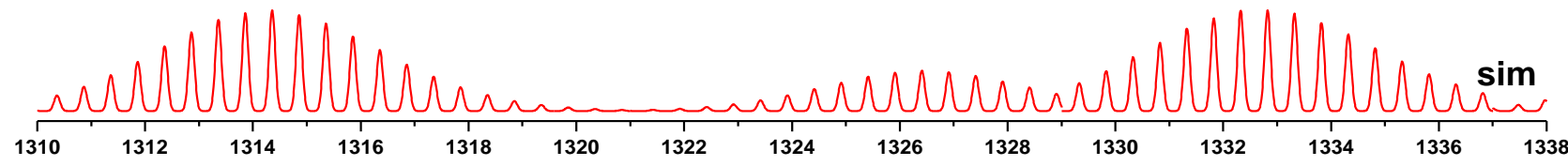

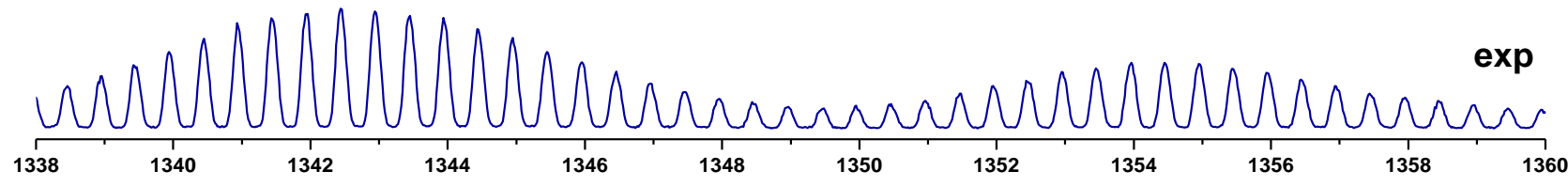

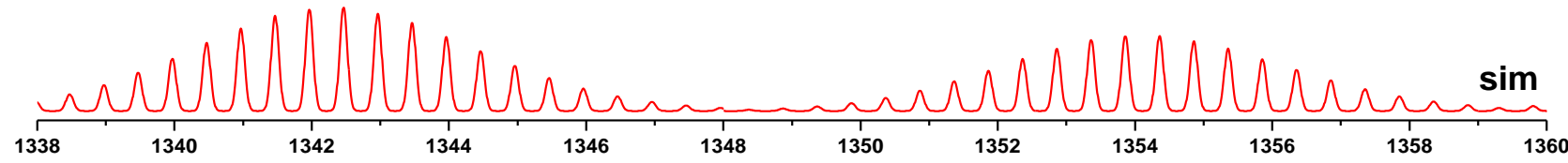

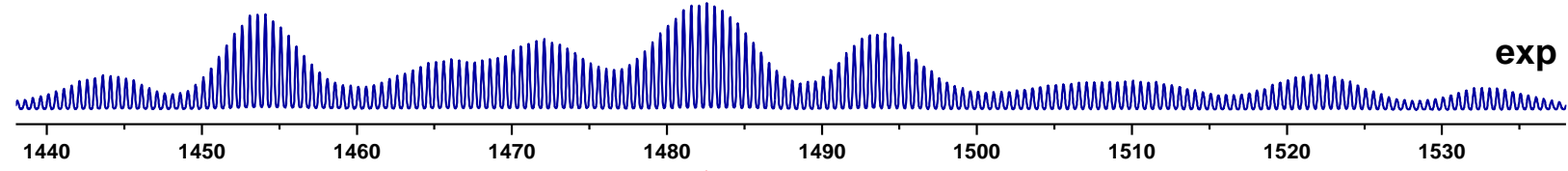

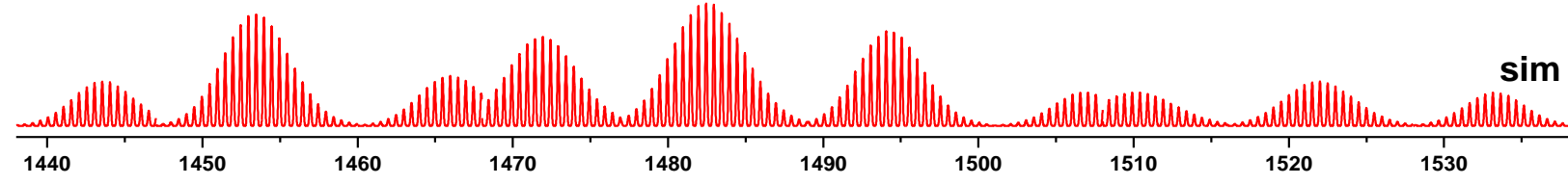

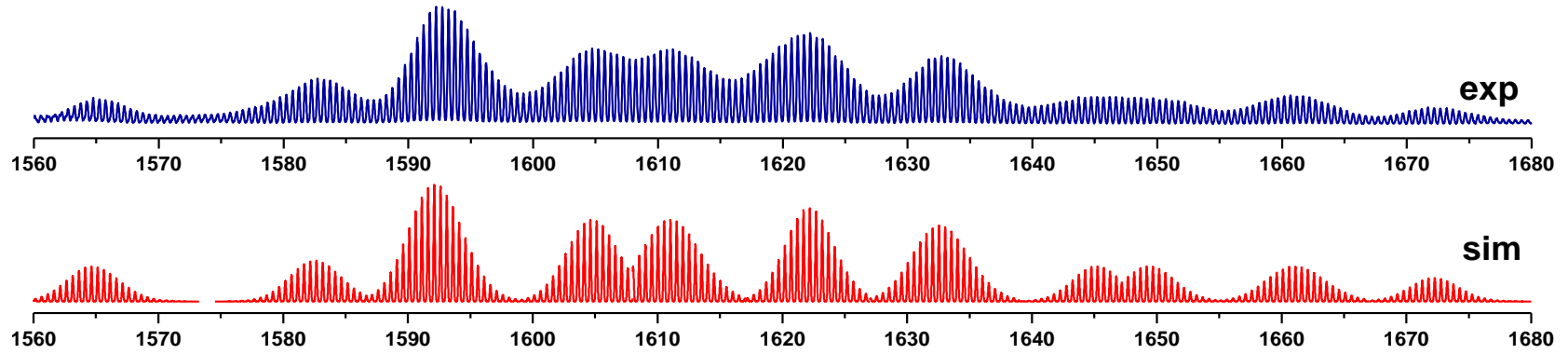



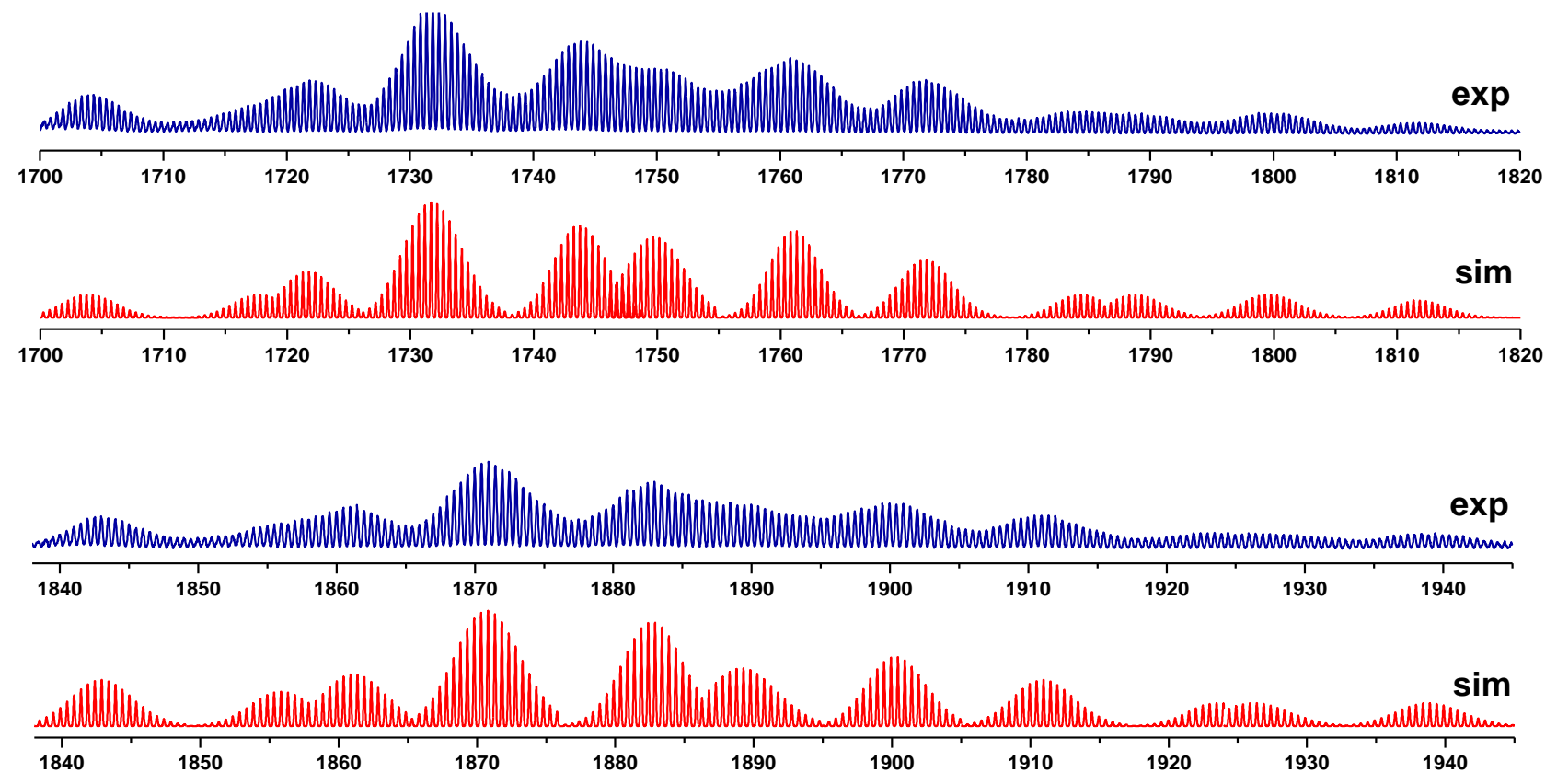

Figure S2. Negative mode ESI-MS of compound 1 in acetonitrile. The envelop assignments are summarized in Table S2.

Table S2. Peak assignments for negative mode ESI-MS of $\mathbf{1}$ in acetonitrile.

\begin{tabular}{|c|c|c|c|c|}
\hline species & $\mathbf{m} / \mathbf{z}($ exp.) & $\mathbf{m} / \mathbf{z}$ (cal.) & charge & envelop assignment \\
\hline $\multirow{5}{*}{\mathrm{Ti}_{18} \mathrm{O}_{27}}^{3-}$ & 795.8688 & 795.8250 & -3 & $\mathrm{Ti}_{18} \mathrm{O}_{27}\left(\mathrm{H}_{2} \mathrm{O}\right)_{11}\left(\mathrm{SO}_{4}\right)_{6} \mathrm{Cl}_{9}$ \\
\hline & 803.8904 & 803.8604 & -3 & $\mathrm{Ti}_{18} \mathrm{O}_{27}\left(\mathrm{H}_{2} \mathrm{O}\right)_{13}(\mathrm{OH})_{2}\left(\mathrm{SO}_{4}\right)_{7} \mathrm{Cl}_{5}$ \\
\hline & 808.5581 & 808.5297 & -3 & $\mathrm{Ti}_{18} \mathrm{O}_{27}\left(\mathrm{H}_{2} \mathrm{O}\right)_{18}(\mathrm{OH})_{2}\left(\mathrm{SO}_{4}\right)_{4} \mathrm{Cl}_{11}$ \\
\hline & 814.5400 & 814.6688 & -3 & $\mathrm{Ti}_{18} \mathrm{O}_{27}\left(\mathrm{H}_{2} \mathrm{O}\right)_{31}(\mathrm{OH})_{11}\left(\mathrm{SO}_{4}\right)_{2} \mathrm{Cl}_{6}$ \\
\hline & 822.5292 & 822.4773 & -3 & $\mathrm{Ti}_{18} \mathrm{O}_{27}\left(\mathrm{H}_{2} \mathrm{O}\right)_{10}\left(\mathrm{SO}_{4}\right)_{6}\left(\mathrm{H}_{2} \mathrm{SO}_{4}\right) \mathrm{Cl}_{9}$ \\
\hline \multirow[t]{7}{*}[\{\mathrm{Ti}_{18}\mathrm{O}_{27}\}+\mathbf{TBAC}]{$^{3-}$} & 887.9507 & 887.9400 & -3 & $\mathrm{Ti}_{18} \mathrm{O}_{27}\left(\mathrm{H}_{2} \mathrm{O}\right)_{13}(\mathrm{OH})_{2}\left(\mathrm{SO}_{4}\right)_{6} \mathrm{Cl}_{7}\left(\mathrm{C}_{16} \mathrm{H}_{36} \mathrm{NCl}\right)$ \\
\hline & 896.6324 & 896.2934 & -3 & $\mathrm{Ti}_{18} \mathrm{O}_{27}\left(\mathrm{H}_{2} \mathrm{O}\right)_{14}(\mathrm{OH})_{3}\left(\mathrm{SO}_{4}\right)_{7} \mathrm{Cl}_{4}\left(\mathrm{C}_{16} \mathrm{H}_{36} \mathrm{NCl}\right)$ \\
\hline & 907.9791 & 908.0115 & -3 & $\mathrm{Ti}_{18} \mathrm{O}_{27}\left(\mathrm{H}_{2} \mathrm{O}\right)_{26}(\mathrm{OH})_{6}\left(\mathrm{SO}_{4}\right)_{2} \mathrm{Cl}_{11}\left(\mathrm{C}_{16} \mathrm{H}_{36} \mathrm{NCl}\right)$ \\
\hline & 914.9713 & 914.6071 & -3 & $\mathrm{Ti}_{18} \mathrm{O}_{27}\left(\mathrm{H}_{2} \mathrm{O}\right)_{15}(\mathrm{OH})\left(\mathrm{SO}_{4}\right)_{7} \mathrm{Cl}_{6}\left(\mathrm{C}_{16} \mathrm{H}_{36} \mathrm{NCl}\right)$ \\
\hline & 923.6107 & 923.5668 & -3 & $\mathrm{Ti}_{18} \mathrm{O}_{27}\left(\mathrm{H}_{2} \mathrm{O}\right)_{10} \mathrm{H}_{2}\left(\mathrm{SO}_{4}\right)_{8} \mathrm{Cl}_{7}\left(\mathrm{C}_{16} \mathrm{H}_{36} \mathrm{NCl}\right)$ \\
\hline & 933.6028 & 933.6481 & -3 & $\mathrm{Ti}_{18} \mathrm{O}_{27}\left(\mathrm{H}_{2} \mathrm{O}\right)_{23}(\mathrm{OH})_{3}\left(\mathrm{SO}_{4}\right)_{5} \mathrm{Cl}_{8}\left(\mathrm{C}_{16} \mathrm{H}_{36} \mathrm{NCl}\right)$ \\
\hline & 941.6165 & 941.5626 & -3 & $\mathrm{Ti}_{18} \mathrm{O}_{27}\left(\mathrm{H}_{2} \mathrm{O}\right)_{11} \mathrm{H}_{3}\left(\mathrm{SO}_{4}\right)_{8} \mathrm{Cl}_{8}\left(\mathrm{C}_{16} \mathrm{H}_{36} \mathrm{NCl}\right)$ \\
\hline $\multirow[t]{10}{*}{\mathrm{Ti}_{18} \mathrm{O}_{27}}^{2-}$ & 1304.4698 & 1304.4649 & -2 & $\mathrm{Ti}_{18} \mathrm{O}_{27}\left(\mathrm{H}_{2} \mathrm{O}\right)_{30}(\mathrm{OH})_{6}\left(\mathrm{SO}_{4}\right)_{7}$ \\
\hline & 1314.4636 & 1314.3577 & -2 & $\mathrm{Ti}_{18} \mathrm{O}_{27}\left(\mathrm{H}_{2} \mathrm{O}\right)_{26}(\mathrm{OH})\left(\mathrm{SO}_{4}\right)_{7} \mathrm{Cl}_{5}$ \\
\hline & 1326.4747 & 1326.4106 & -2 & $\mathrm{Ti}_{18} \mathrm{O}_{27}\left(\mathrm{H}_{2} \mathrm{O}\right)_{28}(\mathrm{OH})_{3}\left(\mathrm{SO}_{4}\right)_{8} \mathrm{Cl}$ \\
\hline & 1332.9487 & 1332.8230 & -2 & $\mathrm{Ti}_{18} \mathrm{O}_{27}\left(\mathrm{H}_{2} \mathrm{O}\right)_{25} \mathrm{H}\left(\mathrm{SO}_{4}\right)_{7} \mathrm{Cl}_{7}$ \\
\hline & 1342.4404 & 1342.4643 & -2 & $\mathrm{Ti}_{18} \mathrm{O}_{27}\left(\mathrm{H}_{2} \mathrm{O}\right)_{36}(\mathrm{OH})_{5}\left(\mathrm{SO}_{4}\right)_{5} \mathrm{Cl}_{5}$ \\
\hline & 1354.4493 & 1354.3582 & -2 & $\mathrm{Ti}_{18} \mathrm{O}_{27}\left(\mathrm{H}_{2} \mathrm{O}\right)_{28}\left(\mathrm{SO}_{4}\right)_{8} \mathrm{Cl}_{4}$ \\
\hline & 1363.9685 & 1363.7962 & -2 & $\mathrm{Ti}_{18} \mathrm{O}_{27}\left(\mathrm{H}_{2} \mathrm{O}\right)_{23} \mathrm{H}_{3}\left(\mathrm{SO}_{4}\right)_{8} \mathrm{Cl}_{7}$ \\
\hline & 1371.9456 & 1371.8918 & -2 & $\mathrm{Ti}_{18} \mathrm{O}_{27}\left(\mathrm{H}_{2} \mathrm{O}\right)_{31}(\mathrm{OH})\left(\mathrm{SO}_{4}\right)_{8} \mathrm{Cl}_{3}$ \\
\hline & 1381.9456 & 1381.8067 & -2 & $\mathrm{Ti}_{18} \mathrm{O}_{27}\left(\mathrm{H}_{2} \mathrm{O}\right)_{25} \mathrm{H}_{3}\left(\mathrm{SO}_{4}\right)_{8} \mathrm{Cl}_{7}$ \\
\hline & 1393.9371 & 1393.8596 & -2 & $\mathrm{Ti}_{18} \mathrm{O}_{27}\left(\mathrm{H}_{2} \mathrm{O}\right)_{29} \mathrm{H}\left(\mathrm{SO}_{4}\right)_{9} \mathrm{Cl}_{3}$ \\
\hline \multirow[t]{7}{*}[\{\mathrm{Ti}_{18}\mathrm{O}_{27}\}+\mathbf{TBAC}]{$^{2-}$} & 1443.6201 & 1443.5469 & -2 & $\mathrm{Ti}_{118} \mathrm{O}_{27}\left(\mathrm{H}_{2} \mathrm{O}\right)_{28}(\mathrm{OH})_{4}\left(\mathrm{SO}_{4}\right)_{7} \mathrm{Cl}_{2}\left(\mathrm{C}_{16} \mathrm{H}_{36} \mathrm{NCl}\right)$ \\
\hline & 1453.5975 & 1453.4619 & -2 & $\mathrm{Ti}_{18} \mathrm{O}_{27}\left(\mathrm{H}_{2} \mathrm{O}\right)_{25}\left(\mathrm{SO}_{4}\right)_{7} \mathrm{Cl}_{6}\left(\mathrm{C}_{16} \mathrm{H}_{36} \mathrm{NCl}\right)$ \\
\hline & 1466.0757 & 1465.9697 & -2 & $\mathrm{Ti}_{18} \mathrm{O}_{27}\left(\mathrm{H}_{2} \mathrm{O}\right)_{25}\left(\mathrm{SO}_{4}\right)_{8} \mathrm{Cl}_{4}\left(\mathrm{C}_{16} \mathrm{H}_{36} \mathrm{NCl}\right)$ \\
\hline & 1472.0909 & 1471.9274 & -2 & $\mathrm{Ti}_{18} \mathrm{O}_{27}\left(\mathrm{H}_{2} \mathrm{O}\right)_{23} \mathrm{H}_{2}\left(\mathrm{SO}_{4}\right)_{7} \mathrm{Cl}_{8}\left(\mathrm{C}_{16} \mathrm{H}_{36} \mathrm{NCl}\right)$ \\
\hline & 1482.5712 & 1482.4560 & -2 & $\mathrm{Ti}_{18} \mathrm{O}_{27}\left(\mathrm{H}_{2} \mathrm{O}\right)_{29} \mathrm{H}\left(\mathrm{SO}_{4}\right)_{5} \mathrm{Cl}_{11}\left(\mathrm{C}_{16} \mathrm{H}_{36} \mathrm{NCl}\right)$ \\
\hline & 1494.0713 & 1494.0539 & -2 & $\mathrm{Ti}_{18} \mathrm{O}_{27}\left(\mathrm{H}_{2} \mathrm{O}\right)_{34}(\mathrm{OH})_{3}\left(\mathrm{SO}_{4}\right)_{6} \mathrm{Cl}_{5}\left(\mathrm{C}_{16} \mathrm{H}_{36} \mathrm{NCl}\right)$ \\
\hline & 1507.0790 & 1507.0611 & -2 & $\mathrm{Ti}_{18} \mathrm{O}_{27}\left(\mathrm{H}_{2} \mathrm{O}\right)_{34}(\mathrm{OH})_{3}\left(\mathrm{SO}_{4}\right)_{7} \mathrm{Cl}_{3}\left(\mathrm{C}_{16} \mathrm{H}_{36} \mathrm{NCl}\right)$ \\
\hline
\end{tabular}




\begin{tabular}{|c|c|c|c|c|}
\hline & 1510.5527 & 1510.4038 & -2 & $\mathrm{Ti}_{18} \mathrm{O}_{27}\left(\mathrm{H}_{2} \mathrm{O}\right)_{26} \mathrm{H}_{4}\left(\mathrm{SO}_{4}\right)_{5} \mathrm{Cl}_{14}\left(\mathrm{C}_{16} \mathrm{H}_{36} \mathrm{NCl}\right)$ \\
\hline & 1522.0580 & 1522.0019 & -2 & $\mathrm{Ti}_{18} \mathrm{O}_{27}\left(\mathrm{H}_{2} \mathrm{O}\right)_{34}\left(\mathrm{SO}_{4}\right)_{6} \mathrm{Cl}_{8}\left(\mathrm{C}_{16} \mathrm{H}_{36} \mathrm{NCl}\right)$ \\
\hline & 1533.0575 & 1533.1451 & -2 & $\mathrm{Ti}_{18} \mathrm{O}_{27}\left(\mathrm{H}_{2} \mathrm{O}\right)_{40}(\mathrm{OH})_{6}\left(\mathrm{SO}_{4}\right)_{7}\left(\mathrm{C}_{16} \mathrm{H}_{36} \mathrm{NCl}\right)$ \\
\hline \multirow{11}{*}[\{\mathrm{Ti}_{18}\mathrm{O}_{27}\}+2\mathrm{TBAC}]{$^{2-}$} & 1564.7502 & 1564.6407 & -2 & $\mathrm{Ti}_{18} \mathrm{O}_{27}\left(\mathrm{H}_{2} \mathrm{O}\right)_{25}(\mathrm{OH})_{3}\left(\mathrm{SO}_{4}\right)_{7} \mathrm{Cl}_{3}\left(\mathrm{C}_{16} \mathrm{H}_{36} \mathrm{NCl}\right)_{2}$ \\
\hline & 1582.7145 & 1582.6735 & -2 & $\mathrm{Ti}_{18} \mathrm{O}_{27}\left(\mathrm{H}_{2} \mathrm{O}\right)_{28}(\mathrm{OH})_{4}\left(\mathrm{SO}_{4}\right)_{7} \mathrm{Cl}_{2}\left(\mathrm{C}_{16} \mathrm{H}_{36} \mathrm{NCl}\right)_{2}$ \\
\hline & 1592.2161 & 1592.1113 & -2 & $\mathrm{Ti}_{18} \mathrm{O}_{27}\left(\mathrm{H}_{2} \mathrm{O}\right)_{26}(\mathrm{OH})\left(\mathrm{SO}_{4}\right)_{7} \mathrm{Cl}_{5}\left(\mathrm{C}_{16} \mathrm{H}_{36} \mathrm{NCl}\right)_{2}$ \\
\hline & 1604.7300 & 1604.6191 & -2 & $\mathrm{Ti}_{18} \mathrm{O}_{27}\left(\mathrm{H}_{2} \mathrm{O}\right)_{26}(\mathrm{OH})\left(\mathrm{SO}_{4}\right)_{8} \mathrm{Cl}_{3}\left(\mathrm{C}_{16} \mathrm{H}_{36} \mathrm{NCl}\right)_{2}$ \\
\hline & 1611.1990 & 1611.0316 & -2 & $\mathrm{Ti}_{18} \mathrm{O}_{27}\left(\mathrm{H}_{2} \mathrm{O}\right)_{21} \mathrm{H}_{3}\left(\mathrm{SO}_{4}\right)_{7} \mathrm{Cl}_{9}\left(\mathrm{C}_{16} \mathrm{H}_{36} \mathrm{NCl}\right)_{2}$ \\
\hline & 1622.1976 & 1622.1970 & -2 & $\mathrm{Ti}_{18} \mathrm{O}_{27}\left(\mathrm{H}_{2} \mathrm{O}\right)_{31}(\mathrm{OH})_{4}\left(\mathrm{SO}_{4}\right)_{8}\left(\mathrm{C}_{16} \mathrm{H}_{36} \mathrm{NCl}\right)_{2}$ \\
\hline & 1632.7024 & 1632.5447 & -2 & $\mathrm{Ti}_{18} \mathrm{O}_{27}\left(\mathrm{H}_{2} \mathrm{O}\right)_{22} \mathrm{H}_{3}\left(\mathrm{SO}_{4}\right)_{8} \mathrm{Cl}_{7}\left(\mathrm{C}_{16} \mathrm{H}_{36} \mathrm{NCl}\right)_{2}$ \\
\hline & 1645.1963 & 1645.2556 & -2 & $\mathrm{Ti}_{18} \mathrm{O}_{27}\left(\mathrm{H}_{2} \mathrm{O}\right)_{37}(\mathrm{OH})_{6}\left(\mathrm{SO}_{4}\right)_{7}\left(\mathrm{C}_{16} \mathrm{H}_{36} \mathrm{NCl}\right)_{2}$ \\
\hline & 1649.1816 & 1649.1906 & -2 & $\mathrm{Ti}_{18} \mathrm{O}_{27}\left(\mathrm{H}_{2} \mathrm{O}\right)_{33}(\mathrm{OH})_{3}\left(\mathrm{SO}_{4}\right)_{8} \mathrm{Cl}\left(\mathrm{C}_{16} \mathrm{H}_{36} \mathrm{NCl}\right)_{2}$ \\
\hline & 1661.1665 & 1661.1061 & -2 & $\mathrm{Ti}_{18} \mathrm{O}_{27}\left(\mathrm{H}_{2} \mathrm{O}\right)_{32} \mathrm{H}\left(\mathrm{SO}_{4}\right)_{6} \mathrm{Cl}_{9}\left(\mathrm{C}_{16} \mathrm{H}_{36} \mathrm{NCl}\right)_{2}$ \\
\hline & 1672.1909 & 1672.2714 & -2 & $\mathrm{Ti}_{18} \mathrm{O}_{27}\left(\mathrm{H}_{2} \mathrm{O}\right)_{40}(\mathrm{OH})_{6}\left(\mathrm{SO}_{4}\right)_{7}\left(\mathrm{C}_{16} \mathrm{H}_{36} \mathrm{NCl}\right)_{2}$ \\
\hline \multirow[t]{12}{*}[\{\mathrm{Ti}_{18}\mathrm{O}_{27}\}+3\mathrm{TBAC}]{$^{2-}$} & 1704.3601 & 1704.2510 & -2 & $\mathrm{Ti}_{18} \mathrm{O}_{27}\left(\mathrm{H}_{2} \mathrm{O}\right)_{24}(\mathrm{OH})_{2}\left(\mathrm{SO}_{4}\right)_{7} \mathrm{Cl}_{4}\left(\mathrm{C}_{16} \mathrm{H}_{36} \mathrm{NCl}\right)_{3}$ \\
\hline & 1717.8878 & 1717.8204 & -2 & $\mathrm{Ti}_{18} \mathrm{O}_{27}\left(\mathrm{H}_{2} \mathrm{O}\right)_{30}(\mathrm{OH})_{5}\left(\mathrm{SO}_{4}\right)_{6} \mathrm{Cl}_{3}\left(\mathrm{C}_{16} \mathrm{H}_{36} \mathrm{NCl}\right)_{3}$ \\
\hline & 1721.8511 & 1721.7777 & -2 & $\mathrm{Ti}_{18} \mathrm{O}_{27}\left(\mathrm{H}_{2} \mathrm{O}\right)_{27}(\mathrm{OH})_{3}\left(\mathrm{SO}_{4}\right)_{7} \mathrm{Cl}_{3}\left(\mathrm{C}_{16} \mathrm{H}_{36} \mathrm{NCl}\right)_{3}$ \\
\hline & 1731.8337 & 1731.6705 & -2 & $\mathrm{Ti}_{18} \mathrm{O}_{27}\left(\mathrm{H}_{2} \mathrm{O}\right)_{21} \mathrm{H}_{2}\left(\mathrm{SO}_{4}\right)_{7} \mathrm{Cl}_{8}\left(\mathrm{C}_{16} \mathrm{H}_{36} \mathrm{NCl}\right)_{3}$ \\
\hline & 1743.8402 & 1743.7455 & -2 & $\mathrm{Ti}_{18} \mathrm{O}_{27}\left(\mathrm{H}_{2} \mathrm{O}\right)_{26}(\mathrm{OH})\left(\mathrm{SO}_{4}\right)_{8} \mathrm{Cl}_{3}\left(\mathrm{C}_{16} \mathrm{H}_{36} \mathrm{NCl}\right)_{3}$ \\
\hline & 1749.8497 & 1749.6810 & -2 & $\mathrm{Ti}_{18} \mathrm{O}_{27}\left(\mathrm{H}_{2} \mathrm{O}\right)_{23} \mathrm{H}_{2}\left(\mathrm{SO}_{4}\right)_{7} \mathrm{Cl}_{8}\left(\mathrm{C}_{16} \mathrm{H}_{36} \mathrm{NCl}\right)_{3}$ \\
\hline & 1761.3296 & 1761.3235 & -2 & $\mathrm{Ti}_{18} \mathrm{O}_{27}\left(\mathrm{H}_{2} \mathrm{O}\right)_{31}(\mathrm{OH})_{4}\left(\mathrm{SO}_{4}\right)_{8}\left(\mathrm{C}_{16} \mathrm{H}_{36} \mathrm{NCl}\right)_{3}$ \\
\hline & 1771.8320 & 1771.8522 & -2 & $\mathrm{Ti}_{18} \mathrm{O}_{27}\left(\mathrm{H}_{2} \mathrm{O}\right)_{36}(\mathrm{OH})_{5}\left(\mathrm{SO}_{4}\right)_{6} \mathrm{Cl}_{3}\left(\mathrm{C}_{16} \mathrm{H}_{36} \mathrm{NCl}\right)_{3}$ \\
\hline & 1784.3093 & 1784.3600 & -2 & $\mathrm{Ti}_{18} \mathrm{O}_{27}\left(\mathrm{H}_{2} \mathrm{O}\right)_{36}(\mathrm{OH})_{5}\left(\mathrm{SO}_{4}\right)_{7} \mathrm{Cl}\left(\mathrm{C}_{16} \mathrm{H}_{36} \mathrm{NCl}\right)_{3}$ \\
\hline & 1788.7933 & 1788.7720 & -2 & $\mathrm{Ti}_{18} \mathrm{O}_{27}\left(\mathrm{H}_{2} \mathrm{O}\right)_{31}(\mathrm{OH})\left(\mathrm{SO}_{4}\right)_{8} \mathrm{Cl}_{3}\left(\mathrm{C}_{16} \mathrm{H}_{36} \mathrm{NCl}\right)_{3}$ \\
\hline & 1799.8048 & 1799.7777 & -2 & $\mathrm{Ti}_{18} \mathrm{O}_{27}\left(\mathrm{H}_{2} \mathrm{O}\right)_{35}(\mathrm{OH})\left(\mathrm{SO}_{4}\right)_{6} \mathrm{Cl}_{7}\left(\mathrm{C}_{16} \mathrm{H}_{36} \mathrm{NCl}\right)_{3}$ \\
\hline & 1811.7828 & 1811.8306 & -2 & $\mathrm{Ti}_{18} \mathrm{O}_{27}\left(\mathrm{H}_{2} \mathrm{O}\right)_{37}(\mathrm{OH})_{3}\left(\mathrm{SO}_{4}\right)_{7} \mathrm{Cl}_{3}\left(\mathrm{C}_{16} \mathrm{H}_{36} \mathrm{NCl}\right)_{3}$ \\
\hline \multirow[t]{11}{*}[\{\mathrm{Ti}_{18}\mathrm{O}_{27}\}+4\mathrm{TBAC}]{$^{2-}$} & 1842.9651 & 1842.8715 & -2 & $\mathrm{Ti}_{18} \mathrm{O}_{27}\left(\mathrm{H}_{2} \mathrm{O}\right)_{24}(\mathrm{OH})_{2}\left(\mathrm{SO}_{4}\right)_{7} \mathrm{Cl}_{4}\left(\mathrm{C}_{16} \mathrm{H}_{36} \mathrm{NCl}\right)_{4}$ \\
\hline & 1855.9922 & 1855.8340 & -2 & $\mathrm{Ti}_{18} \mathrm{O}_{27}\left(\mathrm{H}_{2} \mathrm{O}\right)_{22}\left(\mathrm{SO}_{4}\right)_{8} \mathrm{Cl}_{4}\left(\mathrm{C}_{16} \mathrm{H}_{36} \mathrm{NCl}\right)_{4}$ \\
\hline & 1861.0002 & 1860.8596 & -2 & $\mathrm{Ti}_{18} \mathrm{O}_{27}\left(\mathrm{H}_{2} \mathrm{O}\right)_{25}(\mathrm{OH})\left(\mathrm{SO}_{4}\right)_{7} \mathrm{Cl}_{5}\left(\mathrm{C}_{16} \mathrm{H}_{36} \mathrm{NCl}\right)_{4}$ \\
\hline & 1870.9799 & 1870.7971 & -2 & $\mathrm{Ti}_{18} \mathrm{O}_{27}\left(\mathrm{H}_{2} \mathrm{O}\right)_{21} \mathrm{H}_{2}\left(\mathrm{SO}_{4}\right)_{7} \mathrm{Cl}_{8}\left(\mathrm{C}_{16} \mathrm{H}_{36} \mathrm{NCl}\right)_{4}$ \\
\hline & 1882.9828 & 1882.8721 & -2 & $\mathrm{Ti}_{18} \mathrm{O}_{27}\left(\mathrm{H}_{2} \mathrm{O}\right)_{26}(\mathrm{OH})\left(\mathrm{SO}_{4}\right)_{8} \mathrm{Cl}_{3}\left(\mathrm{C}_{16} \mathrm{H}_{36} \mathrm{NCl}\right)_{4}$ \\
\hline & 1889.4941 & 1889.2624 & -2 & $\mathrm{Ti}_{18} \mathrm{O}_{27}\left(\mathrm{H}_{2} \mathrm{O}\right)_{19} \mathrm{H}_{4}\left(\mathrm{SO}_{4}\right)_{7} \mathrm{Cl}_{10}\left(\mathrm{C}_{16} \mathrm{H}_{36} \mathrm{NCl}\right)_{4}$ \\
\hline & 1900.4668 & 1900.4277 & -2 & $\mathrm{Ti}_{18} \mathrm{O}_{27}\left(\mathrm{H}_{2} \mathrm{O}\right)_{30}(\mathrm{OH})_{3}\left(\mathrm{SO}_{4}\right)_{8} \mathrm{Cl}\left(\mathrm{C}_{16} \mathrm{H}_{36} \mathrm{NCl}\right)_{4}$ \\
\hline & 1910.9730 & 1910.9565 & -2 & $\mathrm{Ti}_{18} \mathrm{O}_{27}\left(\mathrm{H}_{2} \mathrm{O}\right)_{35}(\mathrm{OH})_{4}\left(\mathrm{SO}_{4}\right)_{6} \mathrm{Cl}_{4}\left(\mathrm{C}_{16} \mathrm{H}_{36} \mathrm{NCl}\right)_{4}$ \\
\hline & 1923.4688 & 1923.4641 & -2 & $\mathrm{Ti}_{18} \mathrm{O}_{27}\left(\mathrm{H}_{2} \mathrm{O}\right)_{35}(\mathrm{OH})_{4}\left(\mathrm{SO}_{4}\right)_{7} \mathrm{Cl}_{2}\left(\mathrm{C}_{16} \mathrm{H}_{36} \mathrm{NCl}\right)_{4}$ \\
\hline & 1926.4307 & 1926.3743 & -2 & $\mathrm{Ti}_{18} \mathrm{O}_{27}\left(\mathrm{H}_{2} \mathrm{O}\right)_{34}\left(\mathrm{SO}_{4}\right)_{5} \mathrm{Cl}_{10}\left(\mathrm{C}_{16} \mathrm{H}_{36} \mathrm{NCl}\right)_{4}$ \\
\hline & 1938.8995 & 1938.8821 & -2 & $\mathrm{Ti}_{18} \mathrm{O}_{27}\left(\mathrm{H}_{2} \mathrm{O}\right)_{34}\left(\mathrm{SO}_{4}\right)_{6} \mathrm{Cl}_{8}\left(\mathrm{C}_{16} \mathrm{H}_{36} \mathrm{NCl}\right)_{4}$ \\
\hline
\end{tabular}

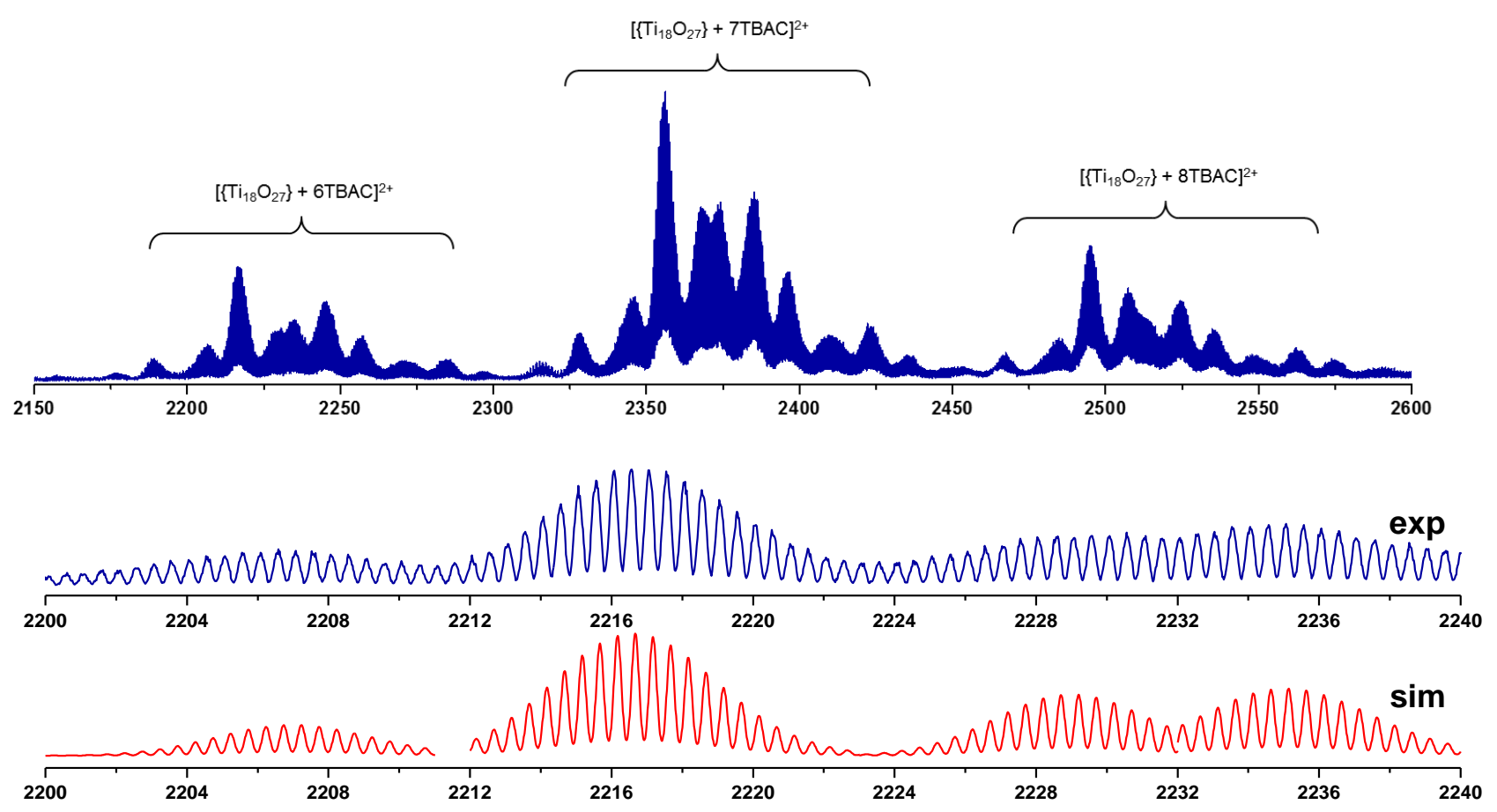


${ }_{2240}$ 2240 ${ }_{2242}^{\prime}{ }_{2244}^{\prime}{ }_{2246}^{\prime}{ }_{2248}$
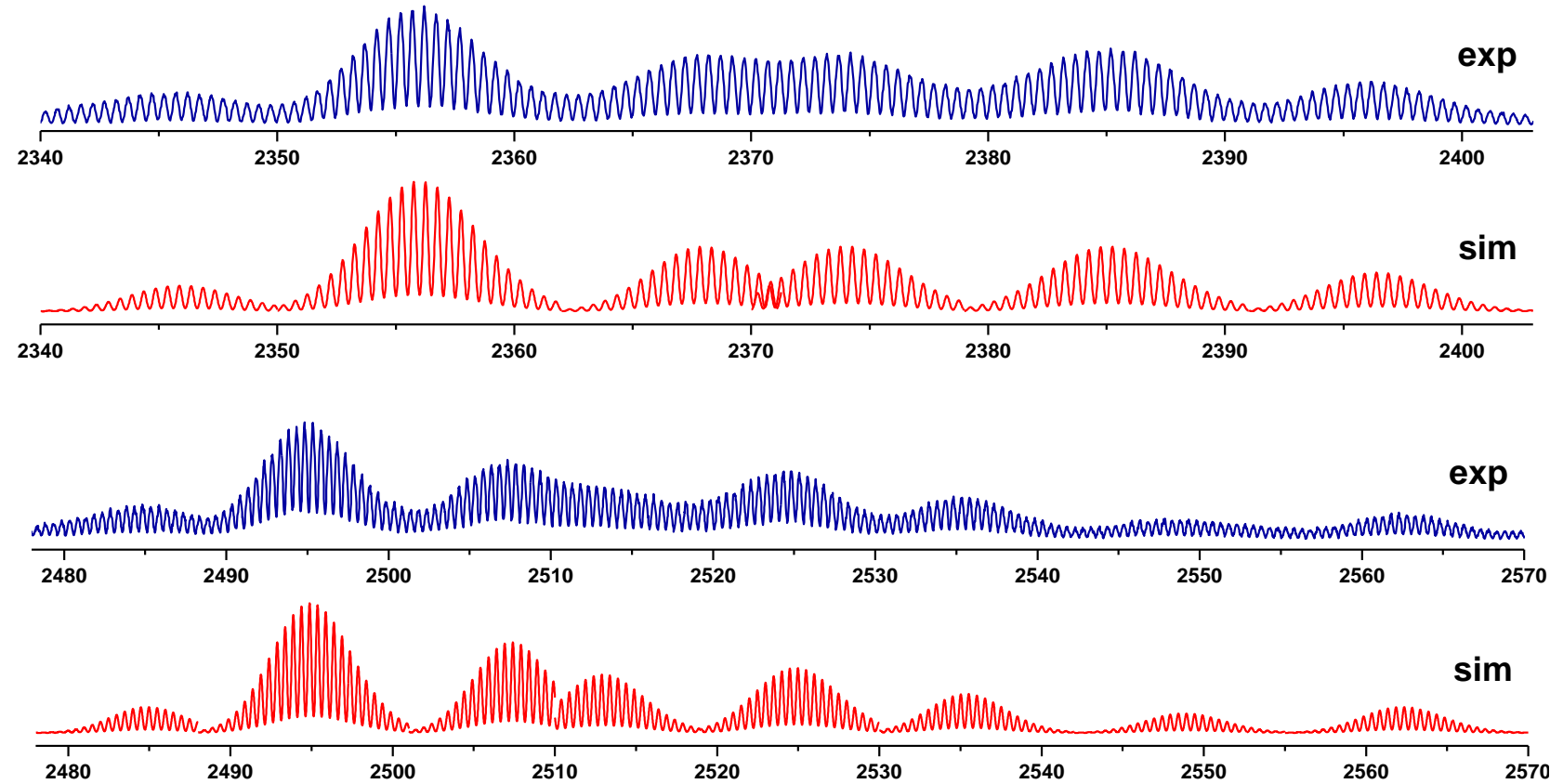

Figure S3. Positive mode ESI-MS of 1 in acetonitrile. The envelop assignments are summarized in Table S3.

Table S3. Peak assignments for positive mode ESI-MS of 1 in acetonitrile

\begin{tabular}{|c|c|c|c|c|}
\hline species & $\mathbf{m} / \mathbf{z}(\exp )$. & $\mathbf{m} / \mathbf{z}$ (cal.) & charge & Envelop assignment \\
\hline $\left.\multirow[t]{8}{*}{\mathrm{Ti}_{18} \mathrm{O}_{27}}+6 \mathrm{TBAC}\right]^{2+}$ & 2189.1865 & 2189.1791 & +2 & $\mathrm{Ti}_{18} \mathrm{O}_{27}\left(\mathrm{H}_{2} \mathrm{O}\right)_{32} \mathrm{H}_{2}\left(\mathrm{SO}_{4}\right)_{8} \mathrm{Cl}_{2}\left(\mathrm{C}_{16} \mathrm{H}_{36} \mathrm{NCl}\right)_{6}$ \\
\hline & 2206.6218 & 2206.7349 & +2 & $\mathrm{Ti}_{18} \mathrm{O}_{27}\left(\mathrm{H}_{2} \mathrm{O}\right)_{38}\left(\mathrm{SO}_{4}\right)_{8}\left(\mathrm{C}_{16} \mathrm{H}_{36} \mathrm{NCl}\right)_{6}$ \\
\hline & 2216.5718 & 2216.6720 & +2 & $\mathrm{Ti}_{18} \mathrm{O}_{27}\left(\mathrm{H}_{2} \mathrm{O}\right)_{33} \mathrm{H}_{3}\left(\mathrm{SO}_{4}\right)_{8} \mathrm{Cl}_{3}\left(\mathrm{C}_{16} \mathrm{H}_{36} \mathrm{NCl}\right)_{6}$ \\
\hline & 2228.5908 & 2228.7027 & +2 & $\mathrm{Ti}_{18} \mathrm{O}_{27}\left(\mathrm{H}_{2} \mathrm{O}\right)_{35} \mathrm{H}_{2}\left(\mathrm{SO}_{4}\right)_{9}\left(\mathrm{C}_{16} \mathrm{H}_{36} \mathrm{NCl}\right)_{6}$ \\
\hline & 2235.0474 & 2235.1376 & +2 & $\mathrm{Ti}_{18} \mathrm{O}_{27}\left(\mathrm{H}_{2} \mathrm{O}\right)_{31} \mathrm{H}_{5}\left(\mathrm{SO}_{4}\right)_{8} \mathrm{Cl}_{5}\left(\mathrm{C}_{16} \mathrm{H}_{36} \mathrm{NCl}\right)_{6}$ \\
\hline & 2245.0195 & 2245.2112 & +2 & $\mathrm{Ti}_{18} \mathrm{O}_{27}\left(\mathrm{H}_{2} \mathrm{O}\right)_{41} \mathrm{H}_{2}\left(\mathrm{SO}_{4}\right)_{6} \mathrm{Cl}_{6}\left(\mathrm{C}_{16} \mathrm{H}_{36} \mathrm{NCl}\right)_{6}$ \\
\hline & 2257.0530 & 2257.1276 & +2 & $\mathrm{Ti}_{18} \mathrm{O}_{27}\left(\mathrm{H}_{2} \mathrm{O}\right)_{30} \mathrm{H}_{6}\left(\mathrm{SO}_{4}\right)_{9} \mathrm{Cl}_{4}\left(\mathrm{C}_{16} \mathrm{H}_{36} \mathrm{NCl}\right)_{6}$ \\
\hline & 2271.5190 & 2271.3398 & +2 & $\mathrm{Ti}_{18} \mathrm{O}_{27}\left(\mathrm{H}_{2} \mathrm{O}\right)_{51}(\mathrm{OH})_{3}\left(\mathrm{SO}_{4}\right)_{6} \mathrm{Cl}\left(\mathrm{C}_{16} \mathrm{H}_{36} \mathrm{NCl}\right)_{6}$ \\
\hline \multirow[t]{6}{*}[\{\mathrm{Ti}_{18}\mathrm{O}_{27}\}+7\mathrm{TBAC}]{$^{2+}$} & 2345.6873 & 2345.8614 & +2 & $\mathrm{Ti}_{18} \mathrm{O}_{27}\left(\mathrm{H}_{2} \mathrm{O}\right)_{38}\left(\mathrm{SO}_{4}\right)_{8}\left(\mathrm{C}_{16} \mathrm{H}_{36} \mathrm{NCl}\right)_{7}$ \\
\hline & 2356.2009 & 2356.2534 & +2 & $\mathrm{Ti}_{18} \mathrm{O}_{27}\left(\mathrm{H}_{2} \mathrm{O}\right)_{29} \mathrm{H}_{5}\left(\mathrm{SO}_{4}\right)_{8} \mathrm{Cl}_{5}\left(\mathrm{C}_{16} \mathrm{H}_{36} \mathrm{NCl}\right)_{7}$ \\
\hline & 2367.7183 & 2367.8293 & +2 & $\mathrm{Ti}_{18} \mathrm{O}_{27}\left(\mathrm{H}_{2} \mathrm{O}\right)_{35} \mathrm{H}_{2}\left(\mathrm{SO}_{4}\right)_{9}\left(\mathrm{C}_{16} \mathrm{H}_{36} \mathrm{NCl}\right)_{7}$ \\
\hline & 2373.7114 & 2373.7645 & +2 & $\mathrm{Ti}_{18} \mathrm{O}_{27}\left(\mathrm{H}_{2} \mathrm{O}\right)_{31} \mathrm{H}_{5}\left(\mathrm{SO}_{4}\right)_{8} \mathrm{Cl}_{5}\left(\mathrm{C}_{16} \mathrm{H}_{36} \mathrm{NCl}\right)_{7}$ \\
\hline & 2385.1860 & 2385.2475 & +2 & $\mathrm{Ti}_{18} \mathrm{O}_{27}\left(\mathrm{H}_{2} \mathrm{O}\right)_{33} \mathrm{H}_{6}\left(\mathrm{SO}_{4}\right)_{6} \mathrm{Cl}_{10}\left(\mathrm{C}_{16} \mathrm{H}_{36} \mathrm{NCl}\right)_{7}$ \\
\hline & 2396.1736 & 2396.3907 & +2 & $\mathrm{Ti}_{18} \mathrm{O}_{27}\left(\mathrm{H}_{2} \mathrm{O}\right)_{45}\left(\mathrm{SO}_{4}\right)_{7} \mathrm{Cl}_{2}\left(\mathrm{C}_{16} \mathrm{H}_{36} \mathrm{NCl}\right)_{7}$ \\
\hline \multirow[t]{7}{*}[\{\mathrm{Ti}_{18}\mathrm{O}_{27}\}+8\mathrm{TBAC}]{$^{2+}$} & 2484.8521 & 2484.9879 & +2 & $\mathrm{Ti}_{18} \mathrm{O}_{27}\left(\mathrm{H}_{2} \mathrm{O}\right)_{38}\left(\mathrm{SO}_{4}\right)_{8}\left(\mathrm{C}_{16} \mathrm{H}_{36} \mathrm{NCl}\right)_{8}$ \\
\hline & 2494.8191 & 2494.8806 & +2 & $\mathrm{Ti}_{18} \mathrm{O}_{27}\left(\mathrm{H}_{2} \mathrm{O}\right)_{29} \mathrm{H}_{5}\left(\mathrm{SO}_{4}\right)_{8} \mathrm{Cl}_{5}\left(\mathrm{C}_{16} \mathrm{H}_{36} \mathrm{NCl}\right)_{8}$ \\
\hline & 2507.3281 & 2507.4106 & +2 & $\mathrm{Ti}_{18} \mathrm{O}_{27}\left(\mathrm{H}_{2} \mathrm{O}\right)_{31} \mathrm{H}_{4}\left(\mathrm{SO}_{4}\right)_{9} \mathrm{Cl}_{2}\left(\mathrm{C}_{16} \mathrm{H}_{36} \mathrm{NCl}\right)_{8}$ \\
\hline & 2512.8149 & 2512.8912 & +2 & $\mathrm{Ti}_{18} \mathrm{O}_{27}\left(\mathrm{H}_{2} \mathrm{O}\right)_{31} \mathrm{H}_{5}\left(\mathrm{SO}_{4}\right)_{8} \mathrm{Cl}_{5}\left(\mathrm{C}_{16} \mathrm{H}_{36} \mathrm{NCl}\right)_{8}$ \\
\hline & 2524.7966 & 2524.9878 & +2 & $\mathrm{Ti}_{18} \mathrm{O}_{27}\left(\mathrm{H}_{2} \mathrm{O}\right)_{46} \mathrm{H}\left(\mathrm{SO}_{4}\right)_{4} \mathrm{Cl}_{9}\left(\mathrm{C}_{16} \mathrm{H}_{36} \mathrm{NCl}\right)_{8}$ \\
\hline & 2535.3508 & 2335.5173 & +2 & $\mathrm{Ti}_{18} \mathrm{O}_{27}\left(\mathrm{H}_{2} \mathrm{O}\right)_{45}\left(\mathrm{SO}_{4}\right)_{7} \mathrm{Cl}_{2}\left(\mathrm{C}_{16} \mathrm{H}_{36} \mathrm{NCl}\right)_{8}$ \\
\hline & 2548.7351 & 2548.9346 & +2 & $\mathrm{Ti}_{18} \mathrm{O}_{27}\left(\mathrm{H}_{2} \mathrm{O}\right)_{37} \mathrm{H}_{4}\left(\mathrm{SO}_{4}\right)_{8} \mathrm{Cl}_{4}\left(\mathrm{C}_{16} \mathrm{H}_{36} \mathrm{NCl}\right)_{8}$ \\
\hline
\end{tabular}



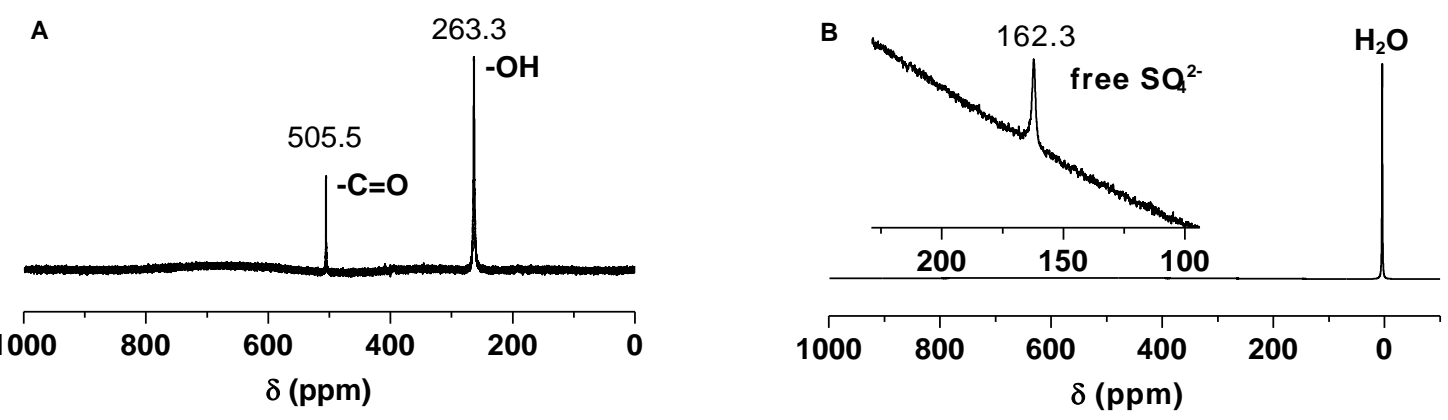

Figure S4. ${ }^{17} \mathrm{O}$ NMR of ${ }^{17} \mathrm{O}$-enriched (A) $\mathrm{CH}_{3} \mathrm{COOH}$ in $\mathrm{MeCN}$ and (B) $\mathrm{SO}_{4}{ }^{2-}$ in water.

\section{Section S3. Vibrational spectra}

The FTIR and the Raman spectra of the solid samples were obtained (Figure S5). The peaks of TBA cations are assigned by comparing with the spectrum of authentic TBAC salt. ${ }^{4}$ The vibrational frequencies of $\mathrm{SO}_{4}$ were assigned by a comparison with that of $\mathrm{TiOSO}_{4}{ }^{5}$ and a literature survey. Hence, the regions of $1000-1300 \mathrm{~cm}^{-1}$ and $400-700 \mathrm{~cm}^{-1}$ were assigned to $v_{3} \& v_{1}$ and $v_{4} \& v_{2}$ of bound $\mathrm{SO}_{4}$, respectively. The strong IR peaks at 839 and $738 \mathrm{~cm}^{-1}$ are assigned to Ti-O bonds, consistent with our previous study. ${ }^{4}$ The two Raman peaks at 208 and 133 $\mathrm{cm}^{-1}$, are tentatively assigned to Ti-O bonds, since anatase $\mathrm{TiO}_{2}$, rutile $\mathrm{TiO}_{2}$, amorphous $\mathrm{TiO}_{2}$, $\left[\mathrm{Ti}_{8} \mathrm{O}_{12}\left(\mathrm{OH}_{2}\right)_{24}\right] \mathrm{Cl}_{8} \cdot \mathrm{nH}_{2} \mathrm{O}$ and $\mathrm{TiOSO}_{4}$ all exhibit strong Ti-O vibrational peaks in the region of $100-250 \mathrm{~cm}^{-1}$.

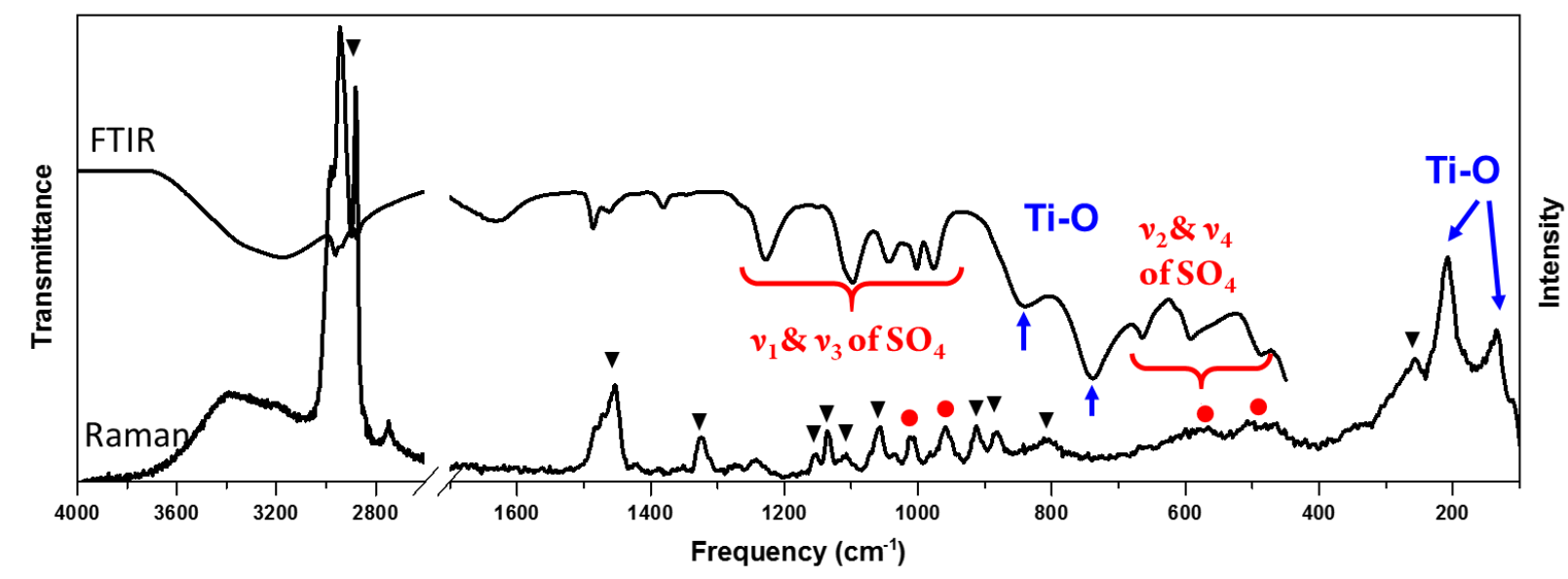

Figure S5. IR (upper) and Raman (lower) spectra of compound 1. The peaks indicated by blue arrows are assigned to vibrational modes of Ti-O bonds, those indicated by "•" are assigned to $\mathrm{SO}_{4}$, and those indicated by " $\nabla$ " are relevant to TBA cations.

4. Zhang, G.; Hou, J.; Li, M.; Tung, C.; Wang, Y., Counteranion-Stabilized Titanium(IV) Isopolyoxocationic Clusters Isolated from Water. Inorg. Chem. 2016, 55, 4704-4709.

5. Zhang, G.; Hou, J.; Tung, C.-H.; Wang, Y., Small Titanium Oxo Clusters: Primary Structures of Titanium(IV) in Water. Inorg. Chem. 2016, 55, 3212-3214. 
Comparing the FTIR of the normal $\mathbf{1}$ and the ${ }^{18} \mathrm{O}$-enriched $\mathbf{1},{ }^{18} \mathrm{O}$-labeling causes substantial red-shift of the 839 and the $738 \mathrm{~cm}^{-1}$ peaks, to $798 \mathrm{~cm}^{-1}$ and to $713 \mathrm{~cm}^{-1}$, respectively. The two bands are assigned to Ti- $\mathrm{O}_{\mu}$ and Ti- $\left(\mathrm{OH}_{2}\right)$, respectively. The peak shift due to isotopic effect is consistent with prediction using the Hook's law. ${ }^{4}$ The Ti-O $\mu$ band is considered to exhibit a higher frequency than Ti- $\left(\mathrm{OH}_{2}\right)$ because it has a shorter Ti-O bond.

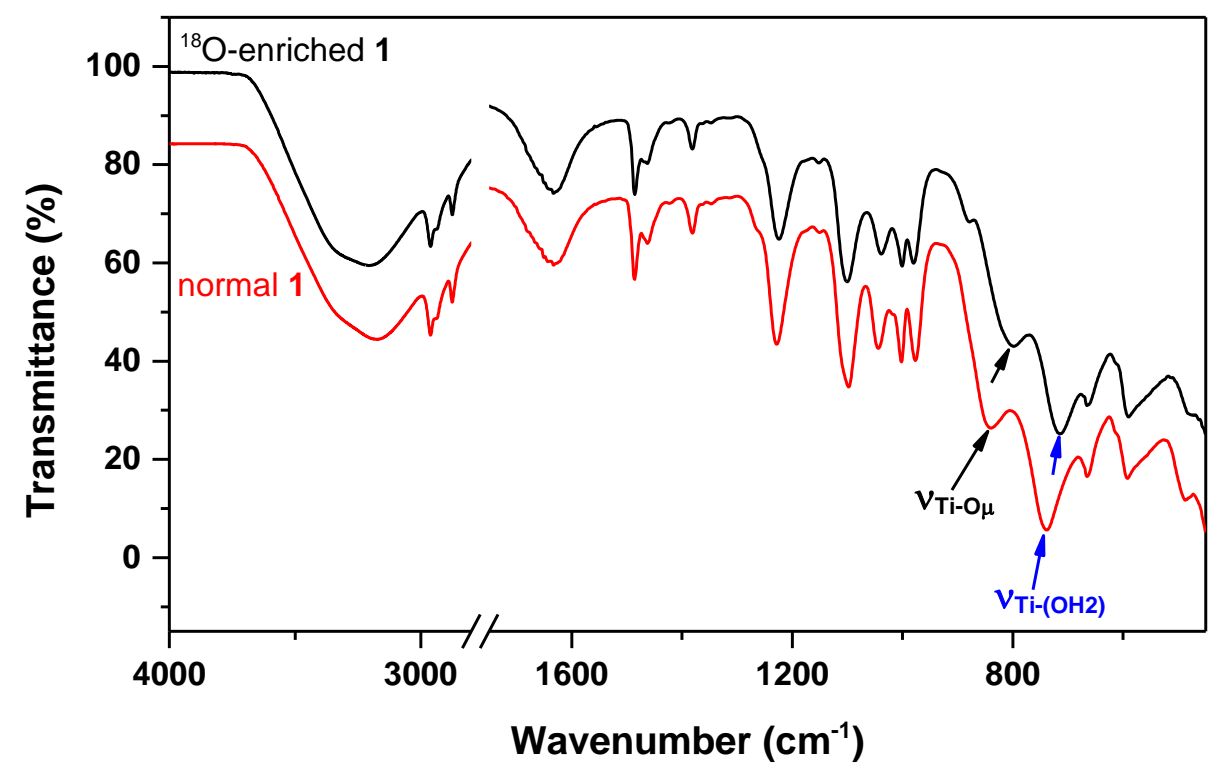

Solution IR were recorded using the ATR-FTIR. Water $(1.0 \mathrm{M} \mathrm{HCl})$ or organic solvents were applied to record the background. For 1 dissolved in $\mathrm{MeCN}$, the IR spectra of the solution sample is reasonably consistent with the authentic solid sample (Figure S6A). Upon solvation, IR frequencies of Ti-O bonds centered at 839 and $738 \mathrm{~cm}^{-1}$ (of the solid sample), are slightly blue-shifted by $10-30 \mathrm{~cm}^{-1}$. The four fundamentals of the $\mathrm{SO}_{4}$ ligands $\left(v_{1}-v_{4}\right)$ were also slightly shifted, meanwhile the peak at $976 \mathrm{~cm}^{-1}$ tentatively assigned to $v_{1}$ of $\mathrm{SO}_{4}$ disappears. Importantly, the IR absorbance of the Ti-O peak at $839 \mathrm{~cm}^{-1}$ and the concentration of 1 follow the Beer-Lambert law (Figure S6, BC). The solution sample, when air-dried, gives a solid whose IR is wellconsistent with that of the authentic solid sample; the slight difference in the peak intensities may be attributed to the low crystallinity of the air-dried sample (panel D). In the Raman spectra, the frequencies of the $\mathrm{SO}_{4}$ ligands in the ranges of $400-600$ and $930-1030 \mathrm{~cm}^{-1}$, and those of Ti-O in the range of $100-250 \mathrm{~cm}^{-1}$, are maintained in acetonitrile solution. Further, the solution vibrational spectra remained unchanged upon aging for a few months under ambient conditions $\left(15-25{ }^{\circ} \mathrm{C}\right)$, indicating the stability of $\mathbf{1}$ in acetonitrile with time. The above evidences, in combination with the ESI-MS and ${ }^{17} \mathrm{O}$ NMR data in the text, strongly indicate the stability of $\mathbf{1}$ in $\mathrm{MeCN}$. Hence vibrational spectroscopy was used as a reliable method to study the solution chemistry in this study. 

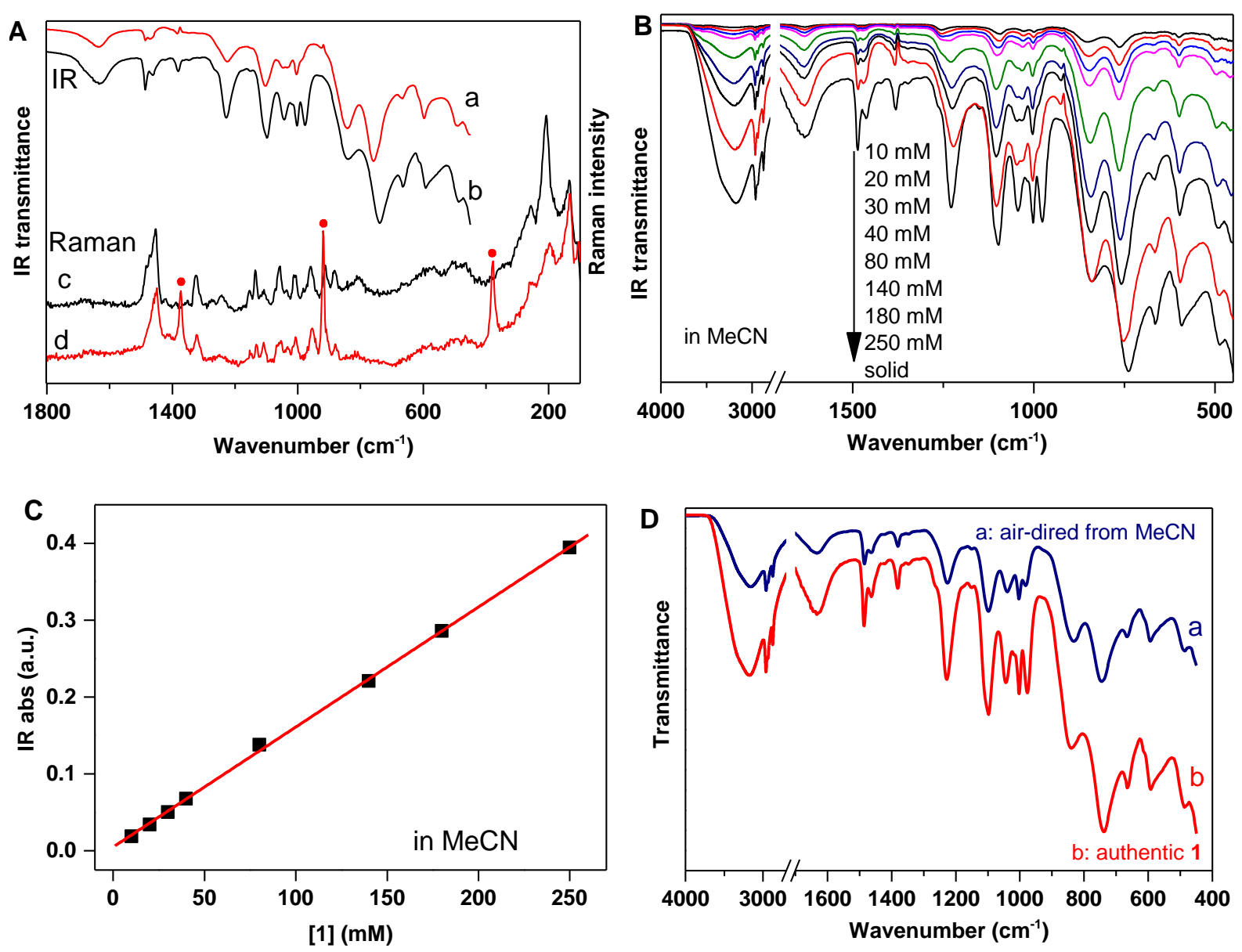

Figure S6. (A) Vibrational spectra of compound 1 in solid forms (curves b and c) and dissolved in MeCN (curves a and d, $180 \mathrm{mM}$ ); (B) the IR spectra of various concentrations of $\mathbf{1}$ in $\mathrm{MeCN}$; (C) IR absorbance at 839 $\mathrm{cm}^{-1}$ as functions of [1]; (D) IR of the air-dried MeCN solution of $\mathbf{1}$. The peaks indicated by the "•" symbol are assigned to the $\mathrm{MeCN}$ solvent.

Due to the relative strong absorption of the solvents (e.g., Figure S7A), some artifacts may exist in the obtained spectra of the solution samples. However, the spectra of 1 in $1.0 \mathrm{M} \mathrm{HCl}$ is reasonably consistent with that of the authentic solid sample (Figure S7B) and that in MeCN (Figure S6A). Data in Figures S7-S8, together with the ${ }^{17} \mathrm{O}$ NMR data in the text, indicate the stability of $\mathbf{1}$ in acidic water.
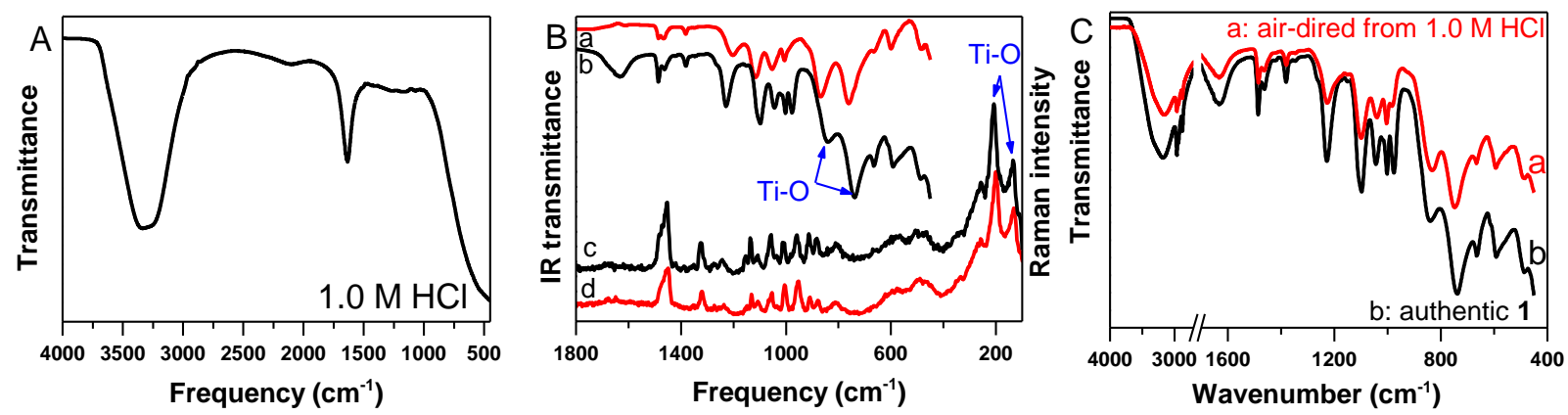

Figure S7. (A) IR of 1.0 M HCl aqueous solution; (B) vibrational spectra of 1 in solid forms (curves b and c) and dissolved in $1.0 \mathrm{M} \mathrm{HCl}$ (curves a and d, $140 \mathrm{mM}$ ); (C) IR of the air-dried $1.0 \mathrm{M} \mathrm{HCl}$ solution of $\mathbf{1}$. 

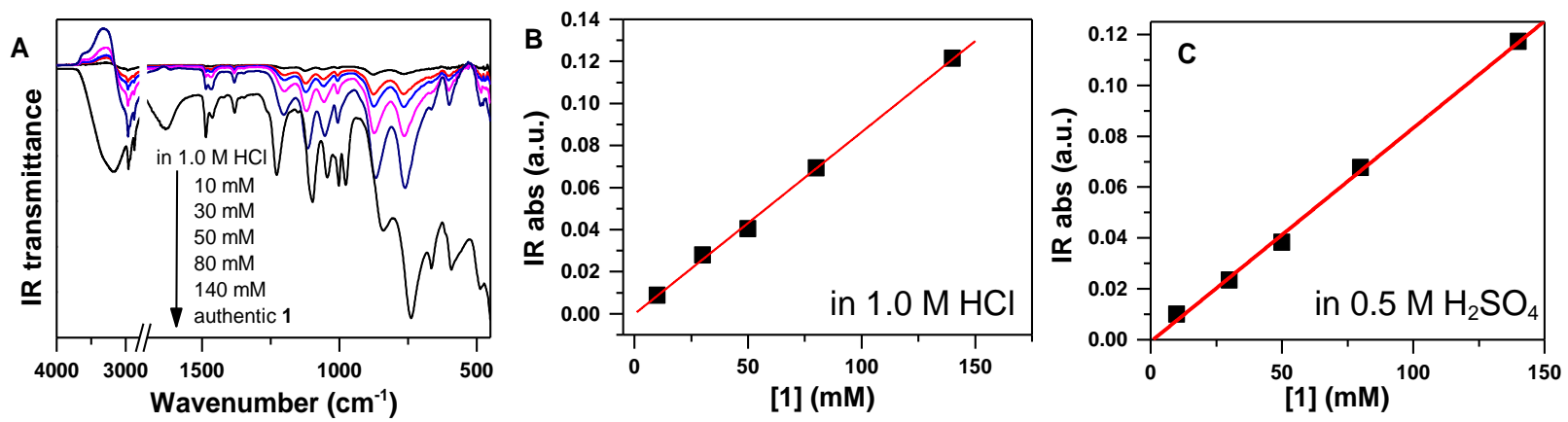

Figure S8. (A) IR of varies concentrations of 1 in $1.0 \mathrm{M} \mathrm{HCl}$; (B) IR absorbance at $868 \mathrm{~cm}^{-1}$ as functions of [1] in $1.0 \mathrm{M} \mathrm{HCl}$; (C) IR absorbance $863 \mathrm{~cm}^{-1}$ as functions of [1] in $0.5 \mathrm{M} \mathrm{H}_{2} \mathrm{SO}_{4}$.

It is interesting to understand what occurs if $\mathbf{1}$ is dissolved directly in neutral water. It was found that upon dissolution of $\mathbf{1}$, the solution becomes acidic and a small amount of white precipitate was produced after $24 \mathrm{~h}$. IR of the solution indicates that the solute is solely $\mathbf{1}$.

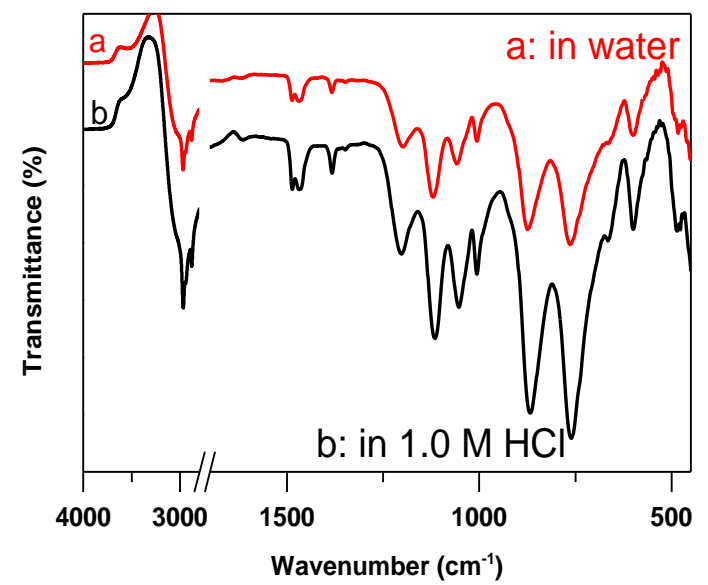

Figure S9. IR of the aqueous solution obtained by dissolution of $\mathbf{1}(80 \mathrm{mM})$ in neutral water. Some precipitate which formed after aging was removed by filtration before the IR measurement.
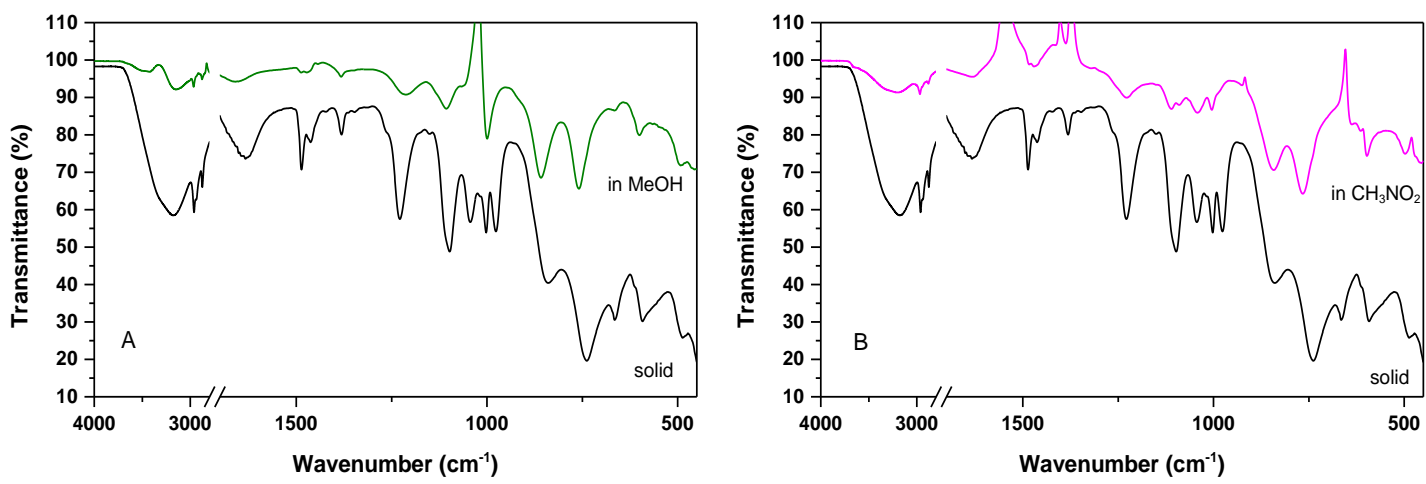

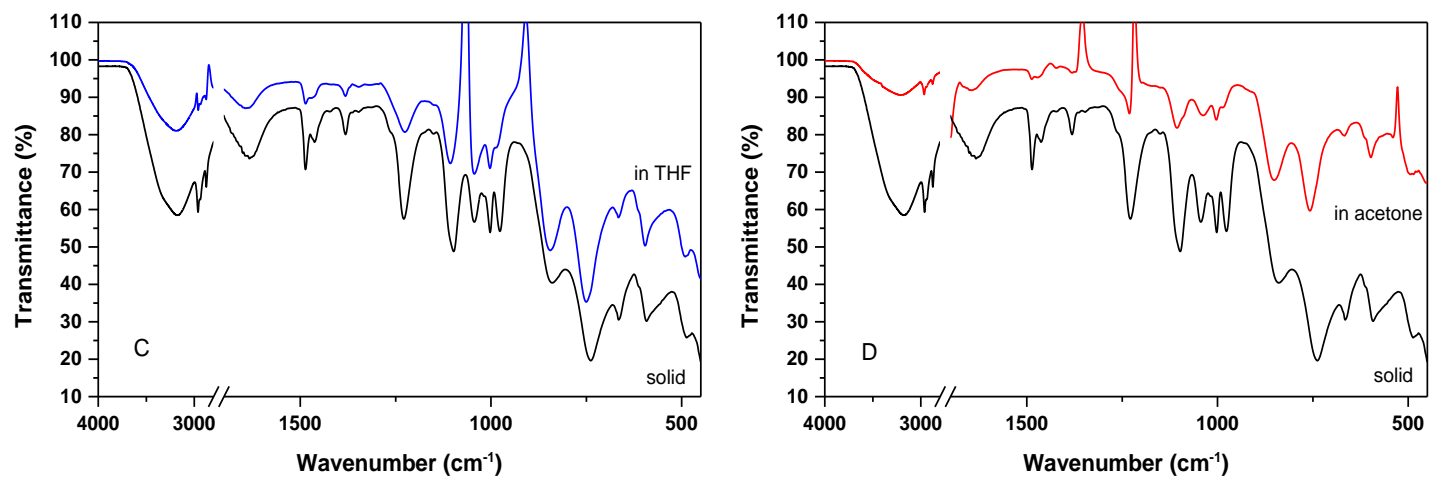

Figure S10. IR spectra of $1(80 \mathrm{mM})$ in methanol, nitromethane, THF and acetone.

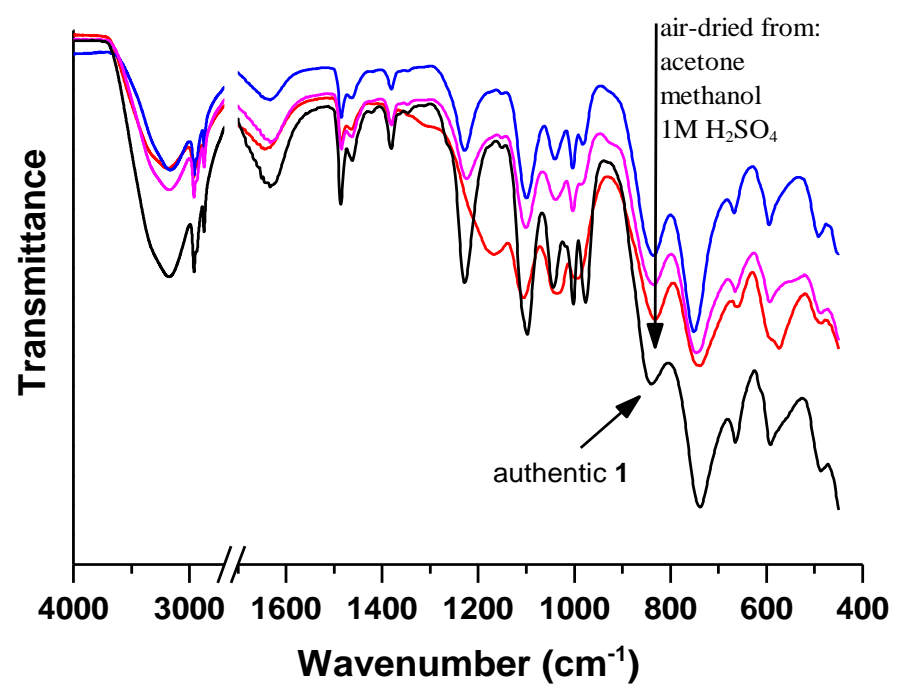

Figure S11. IR of the residue obtained by air-drying of the aged solution samples. These solution samples ( 80 $\mathrm{mM} 1$ ) were aged at room temperature for over a month and air-dried with a fan to give a residue for FTIR measurements.
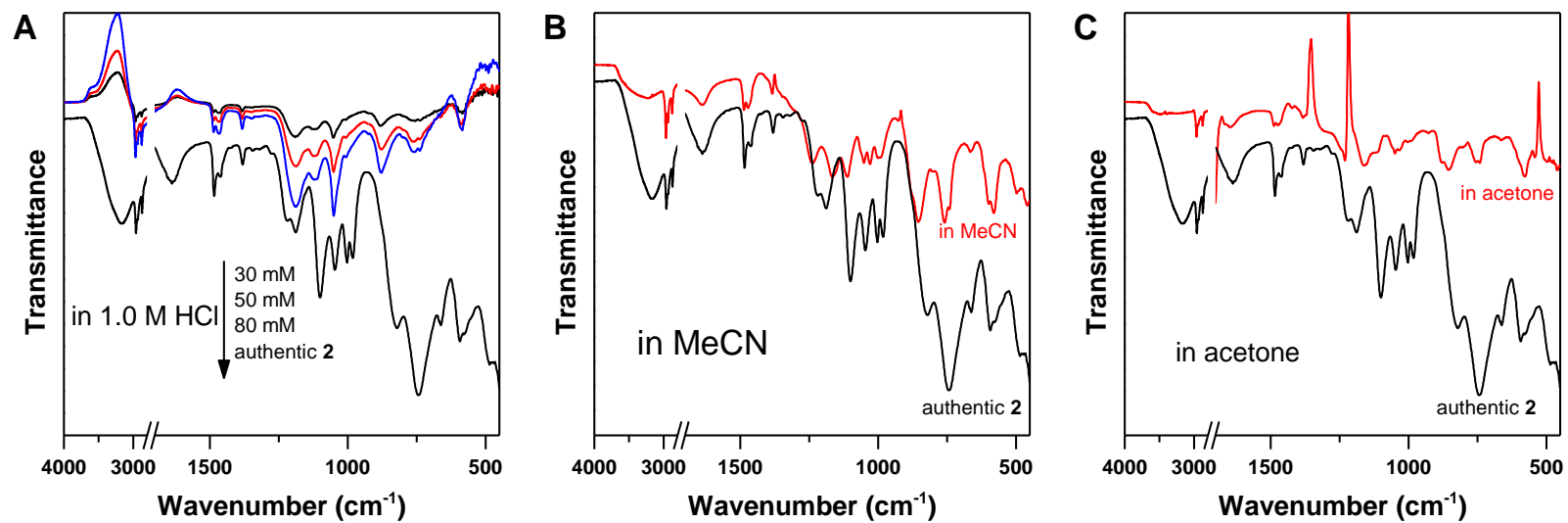

Figure S12. IR of 2 in $1.0 \mathrm{M} \mathrm{HCl}, \mathrm{MeCN}(50 \mathrm{mM})$ and acetone $(30 \mathrm{mM}) .2$ is less soluble in other solvents like methanol, THF, and $\mathrm{CH}_{3} \mathrm{NO}_{2}$. 

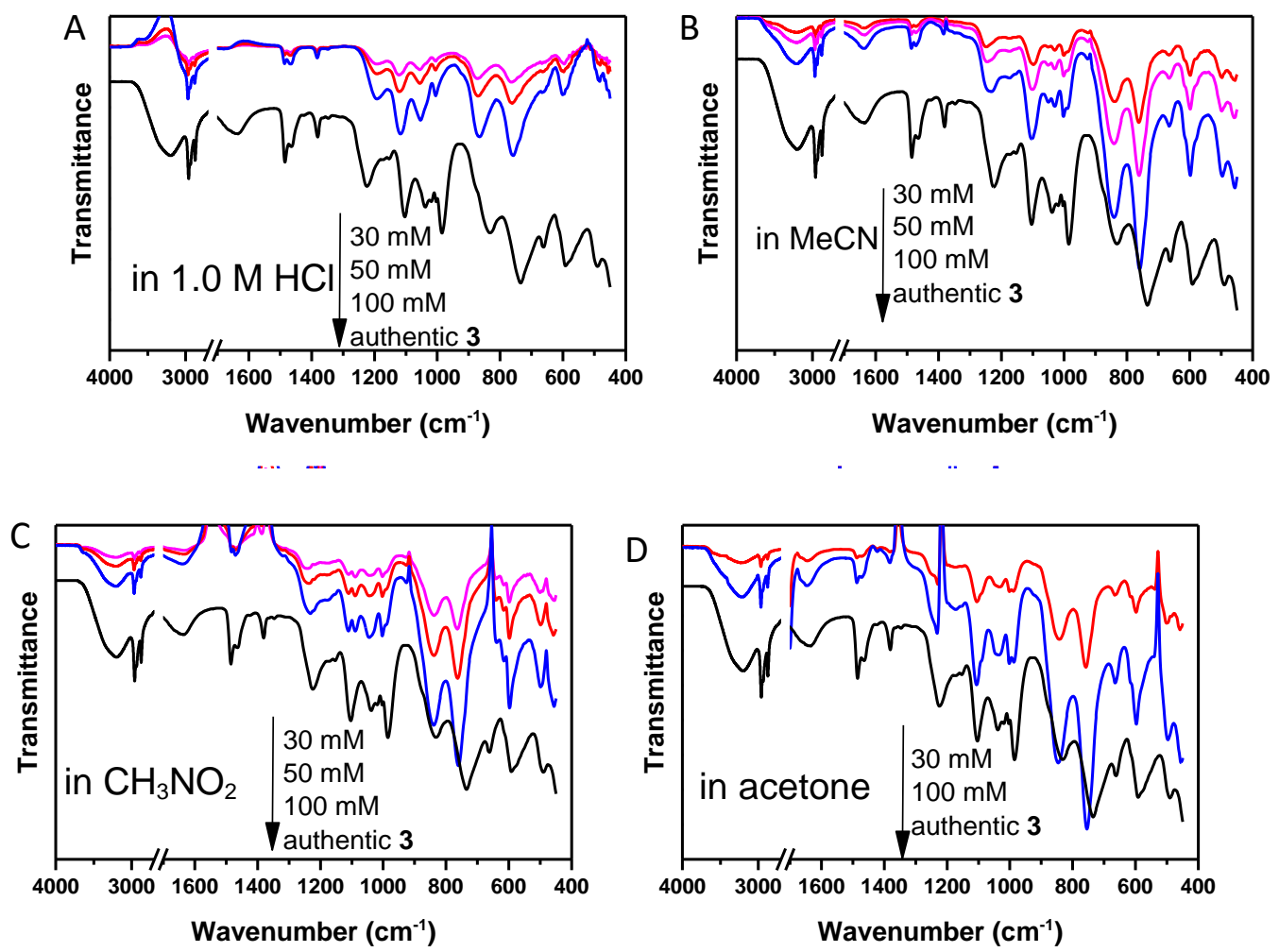

Figure S13. IR of 3 in various solvents. These data suggest that cluster $3 \mathrm{a}$ remains intact in $1.0 \mathrm{M} \mathrm{HCl,} \mathrm{MeCN}$, $\mathrm{CH}_{3} \mathrm{NO}_{2}$ and acetone.
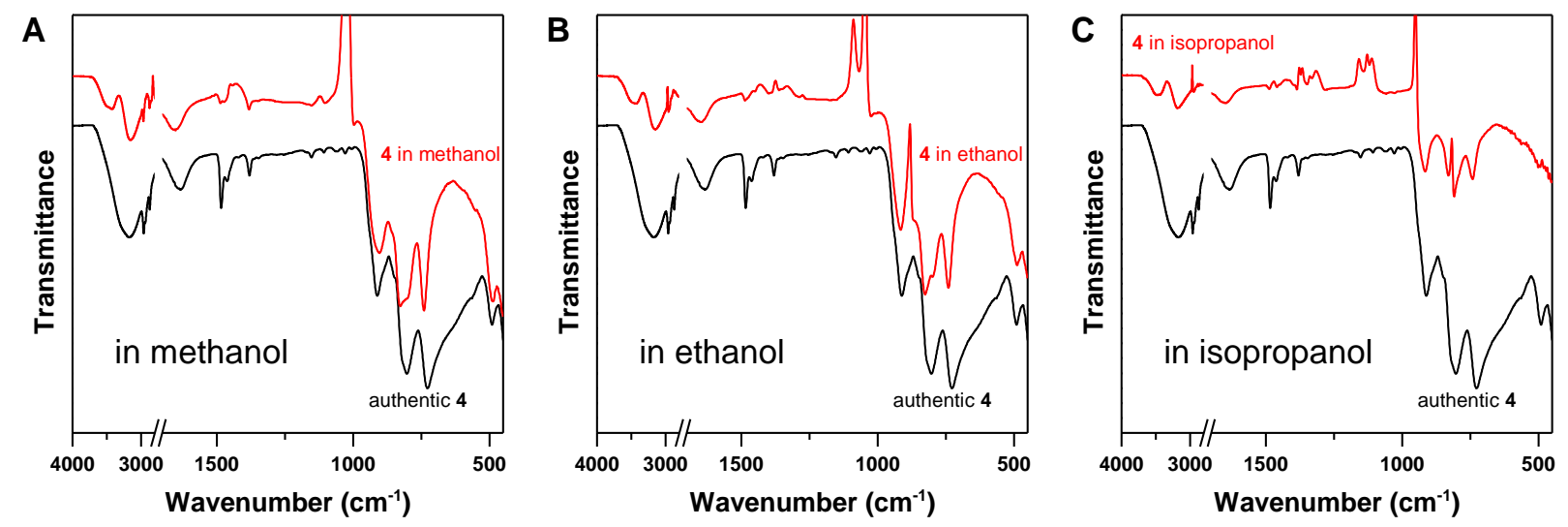

Figure S14. IR of $4(80 \mathrm{mM})$ in various solvents. 


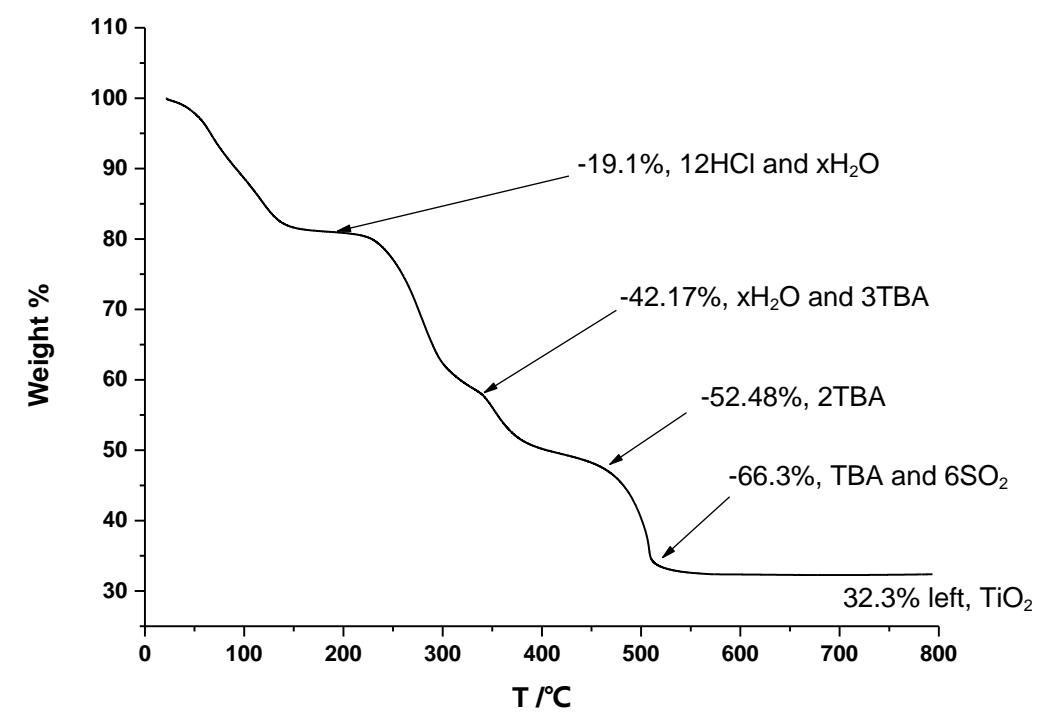

Figure S15. TGA analysis of compound 1 and possible assignments for loss of weight.

\section{Section S4. Functionalization of GO surfaces with 1}

Graphene oxide (GO) was synthesized from natural graphite powder following the modified Hummers method and obtained as an aqueous solution without any surfactant. ${ }^{6}$ The GO surfaces are rich in $-\mathrm{COOH}$ and $-\mathrm{OH}$ moieties. We consider the $\left\{\mathrm{Ti}_{18} \mathrm{O}_{27}\right\}$ cluster may be adsorbed on the hydrophilic GO surfaces via various secondary bonds, like hydrogen bonds between the $\left\{\mathrm{Ti}_{18} \mathrm{O}_{27}\right\}$ aqua ligands and the $-\mathrm{OH}$ moieties of GO. Functionalization of GO with 1 was performed by adding a certain amount of $\mathrm{GO}$ solution to the MeCN solution of $1(80 \mathrm{mM})$, or to the $1.0 \mathrm{M} \mathrm{HCl}$ solution of $\mathbf{1}(80 \mathrm{mM})$. The mixture was stirred for ca. $0.5 \mathrm{~h}$. Both the MeCN and the aqueous solutions could be used to prepare the TEM grids directly. For IR measurements, the mixture was filtered with a $0.22 \mu \mathrm{m}$ Millipore membrane filter to give a wet solid which was dried under vacuum.

High resolution TEM images of the 1-modified GO sample are supplemented. It appears that cluster 1a aggregates may form on the GO surfaces. 

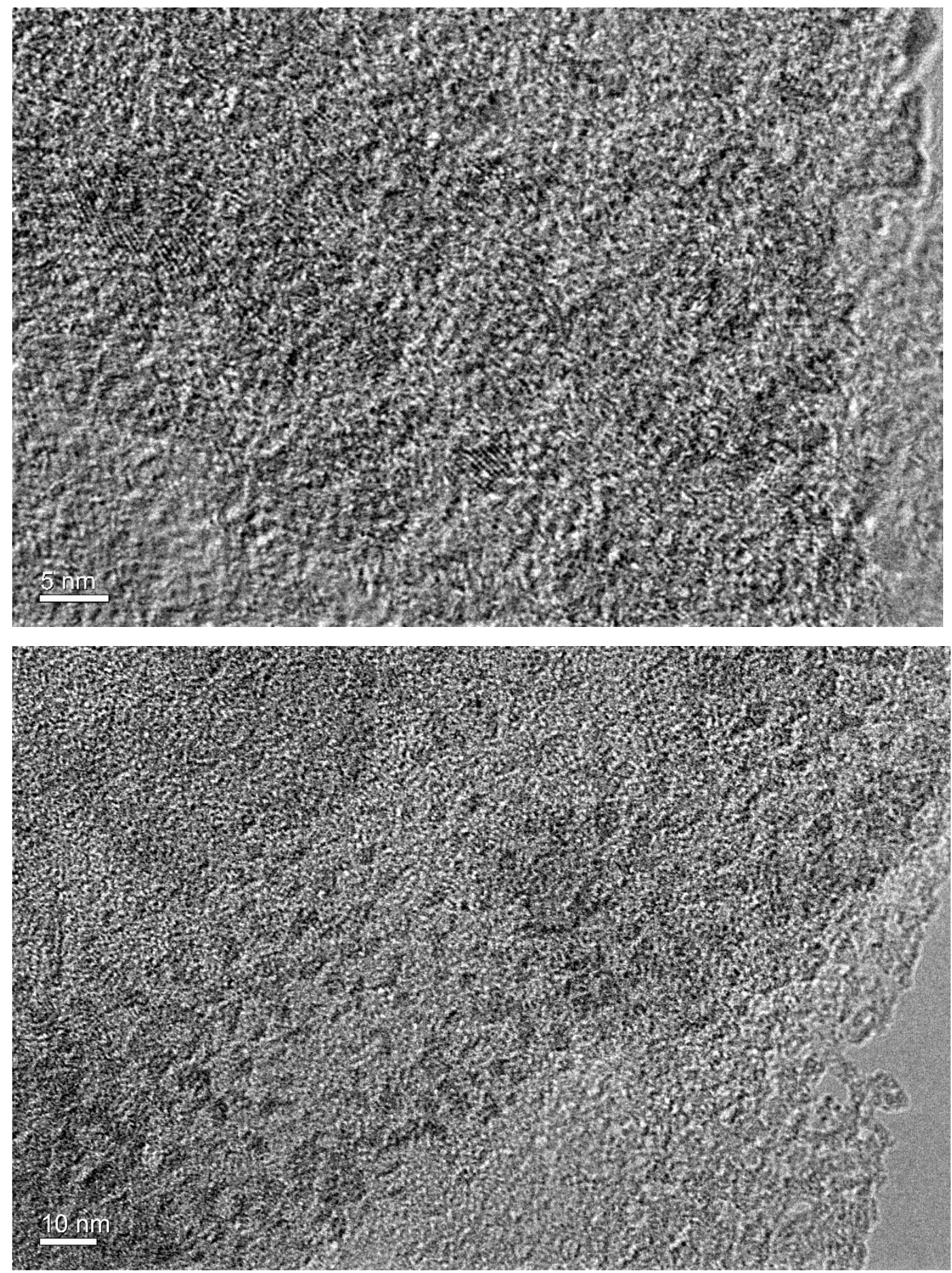

XPS of the $1 / \mathrm{GO}$ sample shown below indicates the atomic ratio of $\mathrm{Ti}, \mathrm{S}$ and $\mathrm{Cl}$ is $3.28: 1.05: 1.51$, consistent with the composition of $\mathbf{1}$. 

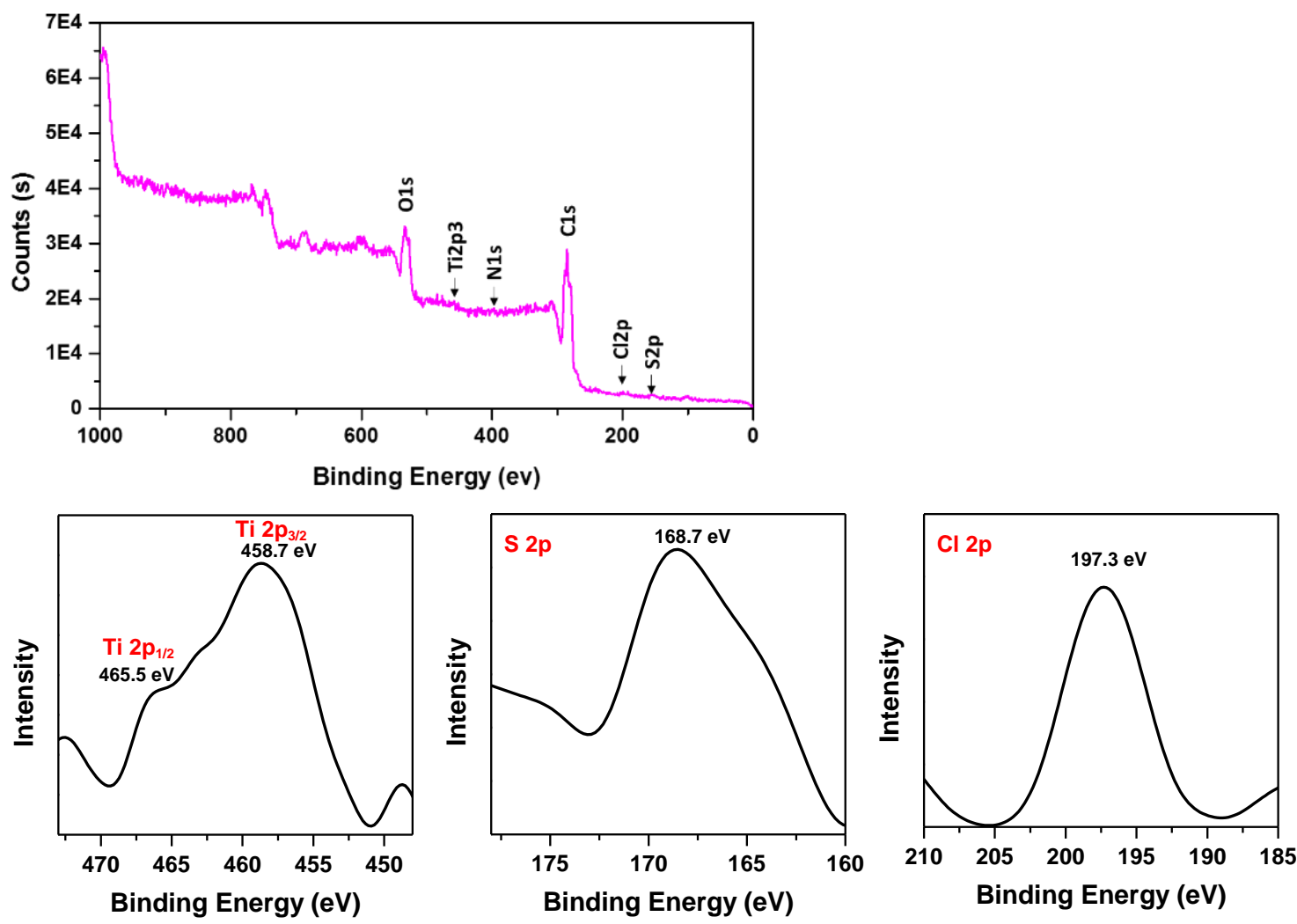

AFM of compound 1 deposited on a planar mica surface was performed. Deposition was done by dipping the mica wafer in a $\mathrm{CH}_{3} \mathrm{CN}$ solution of $20 \mathrm{mM} 1$ and air-drying under ambient conditions. A micrograph $(1.7 \mu \mathrm{m}$ $\times 1.7 \mu \mathrm{m})$ and the height profiles are provided herein. The height of the bright objects is consistent with the dimension of cluster 1a, while the width suggests aggregates of $\mathbf{1}$ formed on the mica surface.
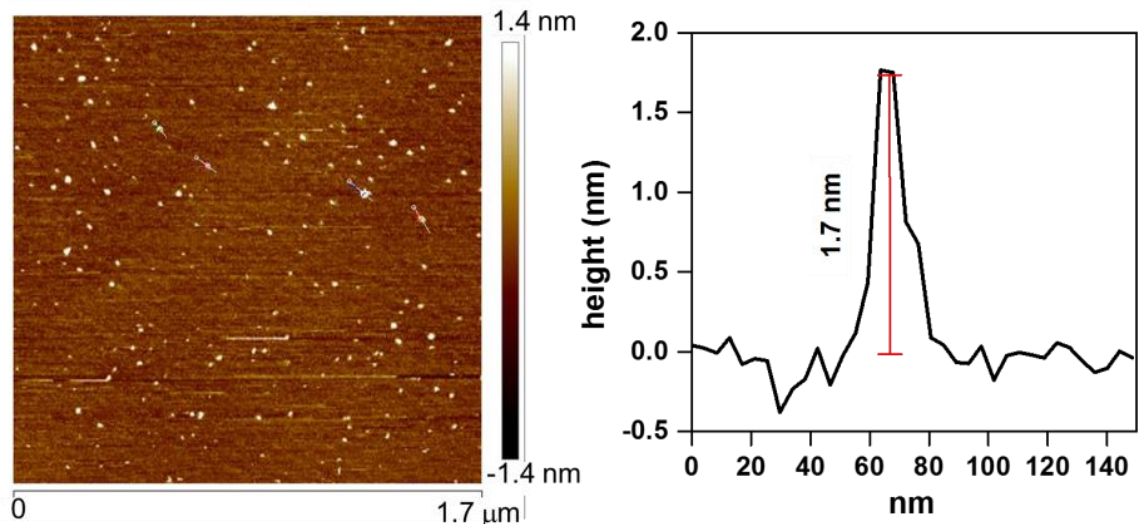

\section{Section S5. UV-vis absorption and diffuse-reflectance spectra}

UV-vis absorption spectra were measured using $1 \mathrm{~cm}$ and $0.2 \mathrm{~cm}$ pathlength cuvettes. This makes it possible to measure the absorbance (at $242 \mathrm{~cm}^{-1}$ ) of solutions up to $10^{-4} \mathrm{M}$ (Figure S16). 

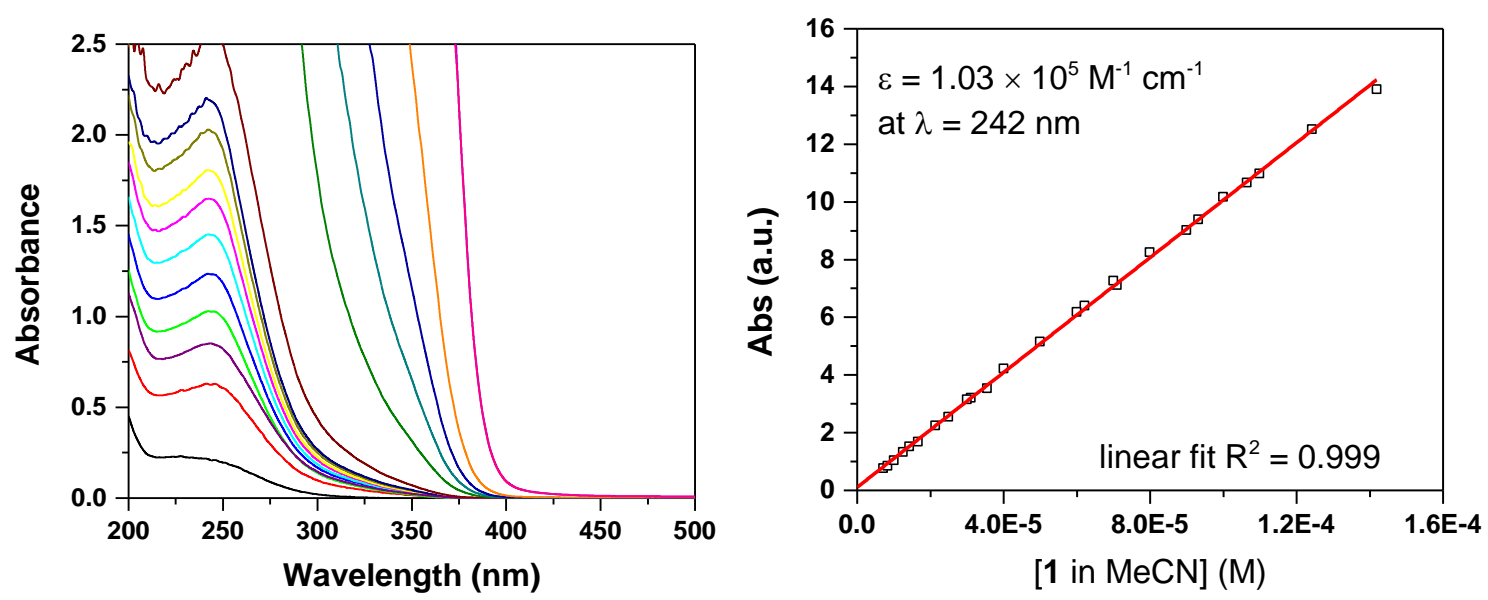

Figure S16. (Left) UV-vis absorption spectra of $1 / \mathrm{MeCN}$ solution and (right) absorbance at $242 \mathrm{~nm}$ as functions of concentration. By linear fitting, the extinction coefficient of $1 / \mathrm{MeCN}$ is $1.03 \times 10^{5} \mathrm{M}^{-1} \mathrm{~cm}^{-1}$ at 242 $\mathrm{nm}$ (the red line in the right panel) and $270 \mathrm{M}^{-1} \mathrm{~cm}^{-1}$ at $380 \mathrm{~nm}$.
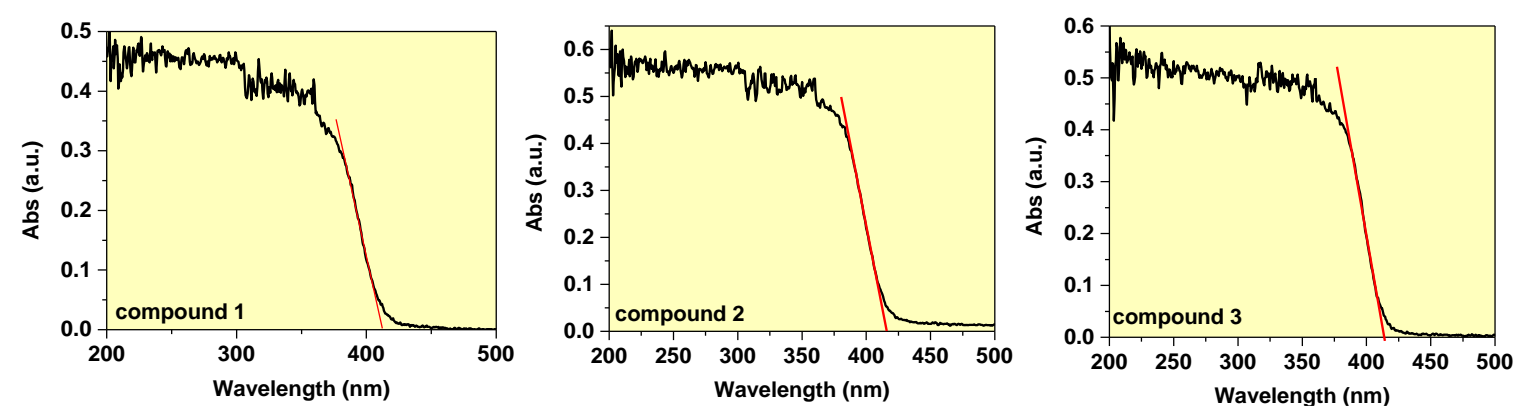

Figure S17. Diffuse reflectance spectra of 1, 2 and 3. The red lines are extrapolation of the linear regions as guidance for the absorption edge.

\section{Section S6. Photocurrent}

$\mathrm{TiO}_{2} /$ FTO film electrode was prepared using the method of Zhao et al. ${ }^{7}$ Functionalization of the $\mathrm{TiO}_{2} / \mathrm{FTO}$ film with 1 was performed by dipping the film in a $\mathrm{CH}_{3} \mathrm{CN}$ solution of $20 \mathrm{mM} 1 \mathrm{for} 5 \mathrm{~min}$, and air-drying under ambient conditions. The photocurrent experiments were performed using a CHI660D workstation and a threeelectrode system, with a Pt plate as the auxiliary electrode and $\mathrm{Ag}$ plate as the reference electrode. A $6 \mathrm{~mL}$ solution of TBAC $(0.5 \mathrm{M})$ in $\mathrm{CH}_{2} \mathrm{Cl}_{2} / \mathrm{HO}^{i} \operatorname{Pr}$ mixture $(5: 1 \mathrm{v}: \mathrm{v})$ was used as the medium. The effective area of the working electrode is $2.12 \mathrm{~cm}^{2}$. A $365 \pm 5 \mathrm{~nm}, 100 \mathrm{~W}$ LED lamp (photon intensity $65 \mathrm{~W} \mathrm{~cm}^{-2}$ ) was employed as the light source. All the photocurrent measurements were performed at room temperature. For IR measurements, the surface-loaded $\mathrm{TiO}_{2}$ was scraped off to give a powder. 1 remained intact during the photocurrent experiments as inferred by the IR measurements. 

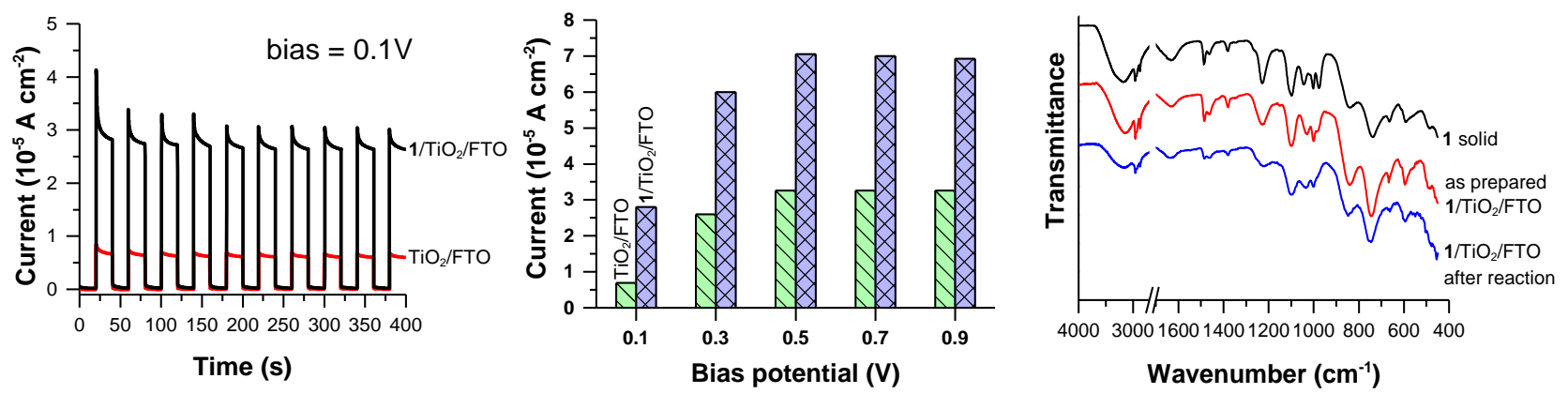

Figure S18. Photocurrent data and IR of the samples.

\section{Section S7. Photocatalytic benzyl alcohol oxidation}

The catalyst $\left(1 ; 6.2 \times 10^{-6} \mathrm{~mol}\right)$ and the substrate (benzyl alcohol; ca. $\left.1 \mathrm{mmol}\right)$ were dissolved in $10.0 \mathrm{~mL}$ of acetonitrile in a vial. The vial was sealed with a butyl rubber stopper and purged with $1.0 \mathrm{~atm} \mathrm{O}_{2}$. Oxidative transformation of benzyl alcohol was initiated by irradiation of the solution with $100 \mathrm{~W} 395 \mathrm{~nm}$ LED at room temperature. An oxygen balloon was used to supply 1.0 atm $\mathrm{O}_{2}$. After every certain time, an aliquot of the solution sample was withdrawn, quantitatively dissolved in $0.2 \mathrm{M} \mathrm{NaOH}$, and filtered with $0.22 \mu \mathrm{m}$ membrane to remove the precipitate. Afterwards, quantification of the solution speciation was performed on an HPLC (Shimadzu LC20;60\% $\mathrm{pH}=2.5 \mathrm{H}_{3} \mathrm{PO}_{4}$ and $40 \%$ acetonitrile as the eluent). The results are shown in Figure S19.
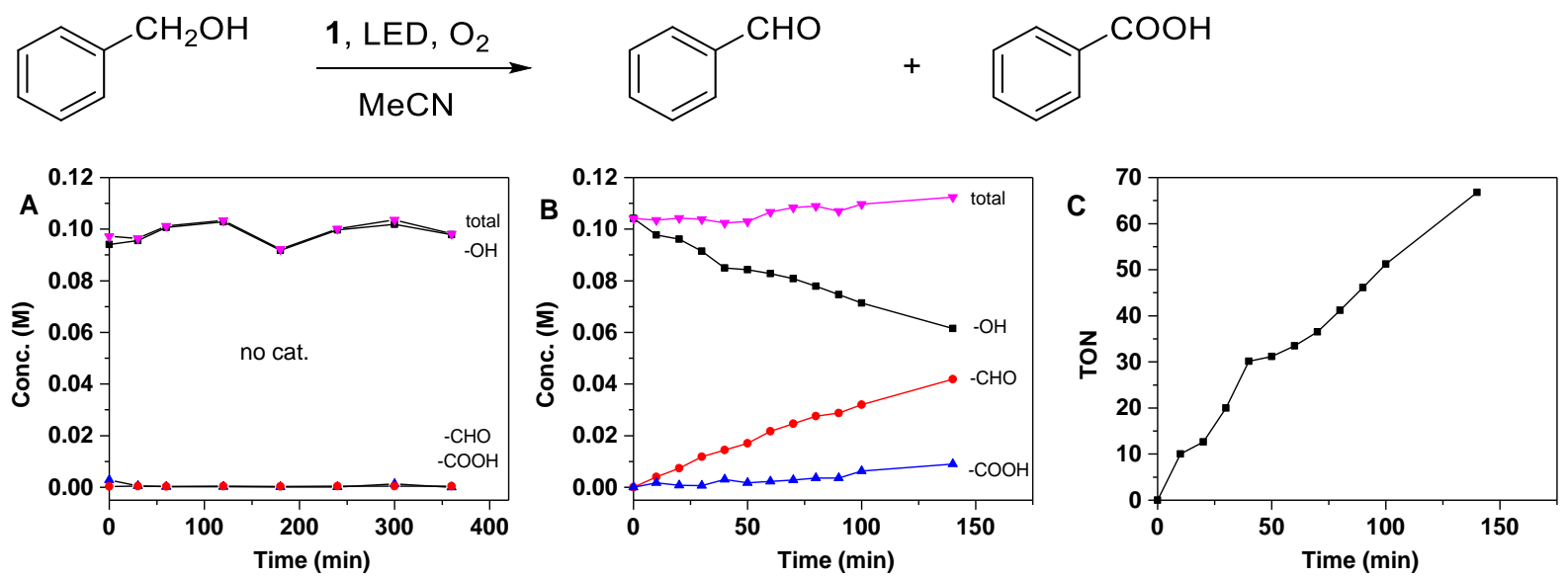

Figure S19. Photocatalytic benzyl alcohol oxidation $(\mathbf{A})$ in the absence of and $(\mathbf{B})$ in the presence of $\mathbf{1}$ under light irradiation; (C) TON variation with time corresponding to panel $\mathbf{B}$. 


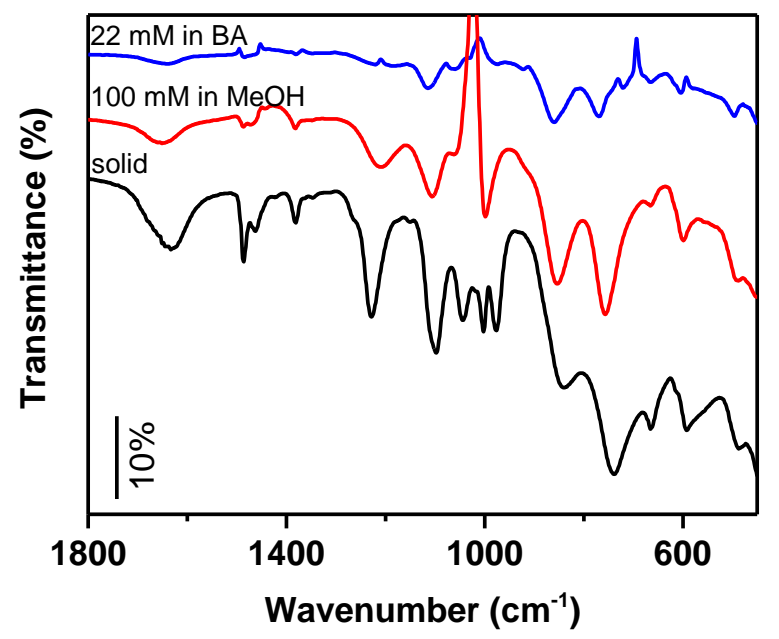

Figure S20. IR spectra of compound 1 in benzyl alcohol (BA), methanol (MeOH) and the authentic spectra. Data suggest that cluster 1a remains intact in benzyl alcohol.

Stability of 1 a during photocatalysis. IR was used to examine if 1a remained intact during photocatalytic oxidations. For high-quality IR, the concentrations of both the substrate (benzyl alcohol) and the catalyst (1) should be relatively large. Hence, $200 \mu \mathrm{L}$ of benzyl alcohol $(960 \mathrm{mM})$ and $30 \mathrm{mM} 1$ were dissolved in $2.0 \mathrm{~mL}$ acetonitrile for the reaction. At certain time upon LED irradiation, the solution sample was taken to measure ATR-FTIR without any pre-treatment (see Figure $\mathrm{S} 21$ ). As can be seen, the growth of the $v(\mathrm{C}=\mathrm{O})$ at ca. 1700 $\mathrm{cm}^{-1}$ shows the formation of benzaldehyde; meanwhile the peak intensity and frequency of the Ti-O bonds (720 $-900 \mathrm{~cm}^{-1}$ ) do not vary. The IR data indicate that cluster 1a is a true photocatalyst and remains intact during the model photocatalytic reaction.

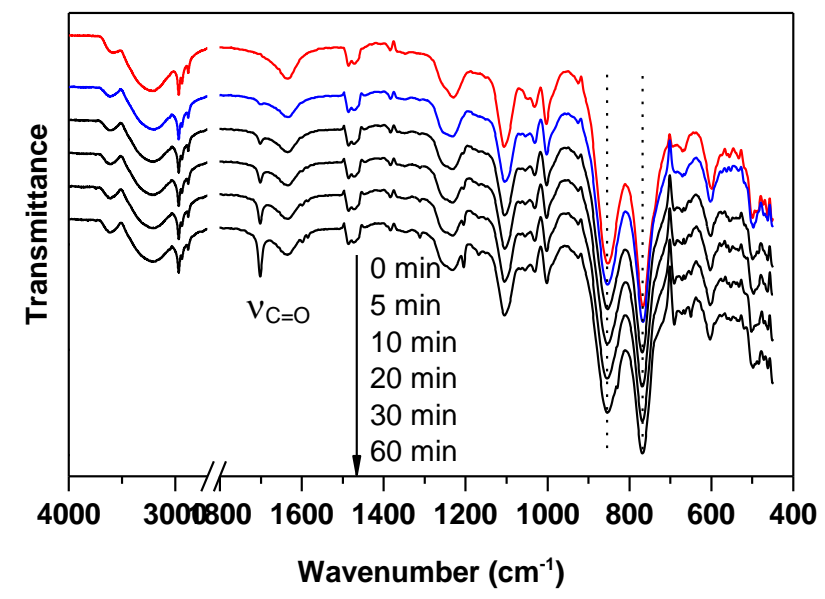

Figure S21. Time-resolved solution IR during photocatalytic oxidation of benzyl alcohol. 